\title{
STRUCTURE FORMATION IN THE SYMMETRON MODEL
}

\author{
Anne-Christine Davis ${ }^{1}$, Baojiu Li ${ }^{1,2}$, David F. Mota ${ }^{3}$, and Hans A. Winther ${ }^{3}$ \\ ${ }^{1}$ DAMTP, Centre for Mathematical Sciences, University of Cambridge, Wilberforce Road, Cambridge CB3 0WA, UK \\ ${ }^{2}$ Kavli Institute for Cosmology Cambridge, Madingley Road, Cambridge CB3 OHA, UK \\ ${ }^{3}$ Institute of Theoretical Astrophysics, University of Oslo, 0315 Oslo, Norway \\ Received 2011 August 18; accepted 2012 January 12; published 2012 March 6
}

\begin{abstract}
Scalar fields, strongly coupled to matter, can be present in nature and still be invisible to local experiments if they are subject to a screening mechanism. The symmetron is one such mechanism that relies on restoration of a spontaneously broken symmetry in regions of high density to shield the scalar fifth force. We have investigated structure formation in the symmetron model by using $N$-body simulations and find observable signatures in both the linear and nonlinear matter power spectrum and on the halo mass function. The mechanism for suppressing the scalar fifth force in high-density regions is also found to work very well.
\end{abstract}

Key words: cosmology: miscellaneous - cosmology: theory - large-scale structure of Universe

Online-only material: color figures

\section{INTRODUCTION}

Our current standard model of cosmology, $\Lambda \mathrm{CDM}$, has been very successful in explaining a large range of observations probing a vast range of length scales. We should nevertheless be open to the possibility that $\Lambda \mathrm{CDM}$ is just a first-order approximation of some more fundamental theory. Many theories of high-energy physics, like string theory and supergravity, predict light gravitationally coupled scalar fields (see, e.g., Binetruy 2006; Linde 2008, and references therein). These scalars may play the role of dark energy (quintessence). If these scalar fields have non-minimal coupling to matter fields, then they could mediate extra forces that are potentially detectable in local experiments.

Over the past few decades, several laboratory and solar system experiments have tried to detect a sign of such fundamental coupled scalar fields (Adelberger 2002; Hoskins et al. 1985; Decca et al. 2007; Bertotti et al. 2003), but the results so far have been negative. Naively, the results of these experiments have ruled out any such scalar fields. However, one should bear in mind that a coupled scalar field might exist but is undetected because of some sort of screening mechanism.

To date we know three types of theoretical mechanisms (see Khoury 2010 for a review) that can explain why such light scalars, if they exist, may not be visible to experiments performed near the Earth. One such class, the chameleon mechanism (Khoury \& Weltman 2004; Brax et al. 2004; Clifton et al. 2005; Mota \& Barrow 2004a, 2004b), operates when the scalars are coupled to matter in such a way that their effective mass depends on the local matter density. In space, where the local mass density is low, the scalars would be light and deviations from general relativity (GR) would be observed. But near the Earth, where experiments are performed, the local mass density is high and the scalar field would acquire a heavy mass, making the interactions short range and therefore unobservable.

The second mechanism, the Vainshtein mechanism (Vainshtein 1972; Deffayet et al. 2002; Arkani-Hamed et al. 2003), operates when the scalar has derivative self-couplings that become important near matter sources such as the Earth. The strong coupling near sources essentially cranks up the kinetic terms, which translates into a weakened matter coupling.
Thus, the scalar screens itself and becomes invisible to experiments. This mechanism is central to the phenomenological viability of braneworld modifications of gravity and galileon scalar theories (Dvali et al. 2000; de Rham et al. 2008; Nicolis et al. 2009; Hinterbichler et al. 2010; Mota et al. 2010; Gabadadze 2009; de Rham 2010; Brax et al. 2011a).

The last mechanism, the one explored in this paper, is the symmetron mechanism (Hinterbichler \& Khoury 2010; Hinterbichler et al. 2011; Olive \& Pospelov 2008; Brax et al. 2011b; Clampitt et al. 2012). In this mechanism, the vacuum expectation value (VEV) of the scalar depends on the local mass density, becoming large in regions of low mass density and small in regions of high mass density. By taking the coupling of the scalar to matter to be proportional to the VEV, we can have a viable theory where the scalar couples with gravitational strength in regions of low density but is decoupled and screened in regions of high density. This is achieved through the interplay of a symmetry breaking potential and a universal quadratic coupling to matter. A similar screening mechanism applies in the case of the environmentally dependent dilaton model (Brax et al. 2010a).

In vacuum, the scalar field acquires a VEV that spontaneously breaks the $\mathbb{Z}_{2}$ symmetry $\phi \rightarrow-\phi$. In the regions of sufficiently high matter density, the field is confined near $\phi=0$, and the symmetry is restored. The fifth force arising from the matter coupling is proportional to $\phi$ making the effects of the scalar small in high-density regions.

In contrast to chameleons, where the strongest constraints (Mota \& Shaw 2006, 2007; Brax et al. 2007a, 2007b, 2008, 2010c; Gannouji et al. 2010; Gies et al. 2008) come from laboratory experiments that in effect wash out any observable effects in the solar system, the symmetron predicts a host of observational signatures in experiments designed to look for deviations from GR, which are just below the current bounds and within reach of the next-generation experiments.

In the simplest formulation (Hinterbichler \& Khoury 2010), which is the one studied here, the symmetron cannot account for dark energy (Hinterbichler et al. 2011). To have a successful cosmology, a cosmological constant must be added to the model. The model is nevertheless a concrete example of a viable modification of gravity that can leave observable imprints 
on cosmological scales. Indeed, the symmetron model was constructed as a scalar-tensor theory with a screening mechanism to suppress fifth forces in solar system tests of GR. Whilst fifth forces are screened in the solar system they could still have observable effects cosmologically. In this paper we address this issue. In particular we investigate the effect the symmetron has on structure formation in order to obtain results that can be compared with observation.

The cosmology of coupled scalar field models is usually strongly constrained by local gravity experiments, which could put limits on the range and the coupling strength of the scalar field. There do exist several cases in which signatures on the linear perturbations are found, but in most cases the range of the field is well below linear scales. To proceed into the region of nonlinear structure formation, one can use the spherical collapse model to obtain the qualitative behavior, but in order to obtain accurate quantitative results deep into the nonlinear regime, one is almost required to perform $N$-body simulations.

Studies of coupled scalar field models, and other models where a fifth-force is present, using $N$-body simulations (Zhao et al. 2010, 2011; Brax et al. 2011c; Li \& Barrow 2011a, 2011b; Li et al. 2011a; Li \& Zhao 2010; Ferraro et al. 2011; Oyaizu et al. 2008; Schmidt et al. 2009; Schmidt 2009; Baldi et al. 2010; Baldi 2009; Hellwing \& Juszkiewicz 2009; Hellwing et al. 2010) have revealed several interesting signatures that can in principle be detected by observations in the near future. For example, in Zhao et al. (2011) and Schmidt (2010) it was found that $f(R)$ theories can give rise to a dependence on the environment of the dynamical to lensing mass ratio of halos; an observable feature that is not found in $\Lambda \mathrm{CDM}$. This signature is also present in the symmetron model (Winther et al. 2011).

In this article we will study the effects a symmetron field has on structure formation. By performing high-resolution $N$-body simulations, we demonstrate explicitly how the symmetron mechanism works in screening the fifth force and obtain observables such as the matter power spectrum and the mass function.

\section{THE SYMMETRON MODEL}

In this section we review the symmetron model, explaining the screening mechanism, and discuss the local constraints on the model parameters. At the end of this section we reparameterize the model parameters by introducing more physically intuitive parameters that will help us discuss the results in the following sections more clearly.

The action governing the dynamics of the symmetron model is given by

$$
\begin{aligned}
S= & \int d x^{4} \sqrt{-g}\left[\frac{R}{2} M_{\mathrm{pl}}^{2}-\frac{1}{2}(\partial \phi)^{2}-V(\phi)\right] \\
& +S_{m}\left(\tilde{g}_{\mu \nu}, \psi_{i}\right),
\end{aligned}
$$

where $g$ is the determinant of the metric $g_{\mu \nu}, R$ is the Ricci scalar, $\psi_{i}$ are the different matter fields, and $M_{\mathrm{pl}} \equiv 1 / \sqrt{8 \pi G}$, where $G$ is the bare gravitational constant. The matter fields couple to the Jordan frame metric $\tilde{g}_{\mu \nu}$ via a conformal rescaling of the Einstein frame metric $g_{\mu \nu}$ given by

$$
\tilde{g}_{\mu \nu}=A^{2}(\phi) g_{\mu \nu}
$$

The coupling function $A(\phi)$ is chosen to be an even polynomial in $\phi$ (to be compatible with the $\phi \rightarrow-\phi$ symmetry)

$$
A(\phi)=1+\frac{1}{2}\left(\frac{\phi}{M}\right)^{2}+\mathcal{O}\left(\frac{\phi^{4}}{M^{4}}\right)
$$

described by a single mass scale $M$. For the range of parameters we are interested in we have $(\phi / M)^{2} \ll 1$. Thus, we can neglect the higher order correction terms. The potential is chosen to be of the symmetry breaking form

$$
V(\phi)=V_{0}-\frac{1}{2} \mu^{2} \phi^{2}+\frac{1}{4} \lambda \phi^{4},
$$

where $V_{0}$ is a cosmological constant (CC). We will for simplicity absorb all contributions to the $\mathrm{CC}$ into $V_{0}$ by simply putting $V_{0} \equiv \Lambda$. We will later see that $\Lambda$ must be taken to be the usual $\mathrm{CC}$ to obtain late time acceleration of the universe. Thus, the symmetron model considered here cannot account for dark energy. It will be interesting to see if a more complicated setup, e.g., extensions of the proposals discussed in Hinterbichler et al. (2011), can lead to dark energy. This is, however, beyond the scope of this work.

The field equation for $\phi$ follows from the variation of the action Equation (1) with respect to $\phi$ and reads

$$
\square \phi=V_{\text {eff, } \phi} .
$$

The effective potential is given in terms of the trace, $T_{m}$, of the matter energy-momentum tensor by

$$
\begin{gathered}
V_{\mathrm{eff}}(\phi)=\frac{1}{2}\left(-\frac{T_{m}}{M^{2}}-\mu^{2}\right) \phi^{2}+\frac{1}{4} \lambda \phi^{4} \\
=\frac{1}{2}\left(\frac{\rho_{m}}{M^{2}}-\mu^{2}\right) \phi^{2}+\frac{1}{4} \lambda \phi^{4}
\end{gathered}
$$

depending on the two mass scales $\mu, M$ and the dimensionless coupling constant $\lambda$. It is convenient to define the critical matter density (and the critical redshift) as

$$
\rho_{\mathrm{SSB}} \equiv \mu^{2} M^{2}=3 H_{0}^{2} M_{\mathrm{pl}}^{2} \Omega_{m}\left(1+z_{\mathrm{SSB}}\right)^{3},
$$

where SSB stands for spontaneous symmetry breaking, $\Omega_{m}$ is the matter density parameter in the universe today, and $H_{0}$ is the Hubble parameter. In regions where $\rho_{m}>\rho_{\mathrm{SSB}}$ (where $\rho_{m}$ is the local matter density) the symmetry $\phi \rightarrow-\phi$ is upheld and the effective potential has a minimum at $\phi_{\min }=0$, whereas in regions where $\rho_{m}<\rho_{\mathrm{SSB}}$ the symmetry is spontaneously broken and the field acquires a VEV

$$
\phi_{\min }= \pm \phi_{0} \sqrt{1-\frac{\rho_{m}}{\rho_{\mathrm{SSB}}}},
$$

where $\phi_{0} \equiv \mu / \sqrt{\lambda}$ is the symmetry breaking VEV for $\rho_{m} \rightarrow 0$. The mass of small fluctuations around the minimum of the effective potential is given by

$$
\begin{aligned}
m_{\phi}^{2} & \equiv V_{\mathrm{eff}, \phi \phi}=\left(\frac{\rho_{m}}{\rho_{\mathrm{SSB}}}-1\right) \mu^{2}+3 \lambda \phi_{\mathrm{min}}^{2} \\
& =\left\{\begin{array}{cl}
\mu^{2}\left(\frac{\rho_{m}}{\rho_{\mathrm{SSB}}}-1\right), & \rho_{m}>\rho_{\mathrm{SSB}} \\
2 \mu^{2}\left(1-\frac{\rho_{m}}{\rho_{\mathrm{SSB}}}\right), & \rho_{m}<\rho_{\mathrm{SSB}} .
\end{array}\right.
\end{aligned}
$$


The symmetron field acquires the longest range, $\lambda_{\phi} \equiv 1 / m_{\phi}$, in low-density regions where

$$
\lambda_{\phi}=\lambda_{0} \equiv \frac{1}{\sqrt{2} \mu} .
$$

For future convenience we introduce the dimensionless quantity $L \equiv \lambda_{0} /\left(\mathrm{Mpc} h^{-1}\right)$, which is the maximum range of the force mediated by the symmetron in units of Mpc $h^{-1}$.

The gravitational field equation for $g_{\mu \nu}$ is given by

$$
G_{\mu \nu}=8 \pi G T_{\mu \nu}
$$

where the total energy-momentum tensor $T_{\mu \nu}$ is the sum of the matter and scalar field parts:

$$
T_{\mu \nu}=A(\phi) T_{\mu \nu}^{m}+\phi_{; \mu} \phi_{; \nu}-g_{\mu \nu}\left(\frac{1}{2}(\partial \phi)^{2}+V(\phi)\right) .
$$

Note that the matter part itself is not conserved, but instead satisfies

$$
\nabla_{\nu} T_{m}^{\mu \nu}=\frac{d \log A(\phi)}{d \phi}\left(T_{m} \nabla^{\mu} \phi-T_{m}^{\mu \nu} \nabla_{\nu} \phi\right) .
$$

In $N$-body simulations we are interested in describing the matter sector by particles. The energy-momentum tensor of an individual particle with mass $m_{0}$ at position $\mathbf{r}_{\mathbf{0}}$ is given by

$$
T_{m}^{\mu \nu}(\mathbf{r})=\frac{m_{0}}{\sqrt{-g}} \delta\left(\mathbf{r}-\mathbf{r}_{0}\right) \dot{r}_{0}^{\mu} \dot{r}_{0}^{v},
$$

where $\mathbf{r}$ is the general spatial coordinate. Taking the divergence of Equation (12) and using the Bianchi identity, we get the geodesic equation for the matter particles

$$
\ddot{r}_{0}^{\mu}+\Gamma_{\alpha \gamma}^{\mu} r_{0}^{\alpha} r_{0}^{\gamma}=-\frac{d \log A(\phi)}{d \phi}\left(\nabla^{\mu} \phi+\dot{\phi} \dot{r}_{0}^{\mu}\right)
$$

which for $A \equiv 1$ reduces to the standard geodesic equation in GR.

From Equation (16) we see that the symmetron field gives rise to a fifth force on the matter fields that, in the nonrelativistic limit, is given by

$$
\vec{F}_{\phi}=\frac{\phi}{M^{2}} \vec{\nabla} \phi=\frac{\beta}{M_{\mathrm{pl}}}\left(\frac{\phi}{\phi_{0}}\right) \vec{\nabla} \phi
$$

where we have introduced the coupling constant $\beta \equiv$ $\phi_{0} M_{\mathrm{pl}} / M^{2}$.

The static, spherically symmetric solutions of the field equations were found in Hinterbichler \& Khoury (2010). For two test masses in a region where $\phi=\phi_{B}$ it was shown that the fifth force is simply

$$
\frac{F_{\phi}}{F_{N}}=2 \beta^{2}\left(\frac{\phi_{B}}{\phi_{0}}\right)^{2}
$$

In a low-density region $\left(\rho \ll \rho_{\mathrm{SSB}}\right)$ we have $\phi_{B}=\phi_{0}$ and the fifth force is comparable with gravity for $\beta=\mathcal{O}(1)$.

For very large bodies, the situation is quite different. The symmetry is restored in the interior of the body and the fifth force on a test mass outside becomes

$$
\frac{F_{\phi}}{F_{N}}=2 \beta^{2}\left(\frac{\phi_{B}}{\phi_{0}}\right)^{2} \frac{1}{\alpha}, \alpha^{-1}=2 \frac{\rho_{\mathrm{SSB}}}{\rho_{\text {body }}}\left(\frac{\lambda_{0}}{R_{\text {body }}}\right)^{2} .
$$

The fifth force is suppressed by a factor $\alpha^{-1} \ll 1-$ similar to the thin shell factor found in chameleon theories (Khoury \& Weltman 2004).

We also see that if the test masses are inside a screened region $\left(\phi_{B} / \phi_{0} \ll 1\right)$ the force will be further suppressed.

Since the field is long ranged (and universally coupled) in almost all situations today, the theory is best constrained by solar system experiments that have been performed with high precision.

It turns out that as long as our Galaxy is sufficiently screened (10 $\left.\lesssim \alpha_{G}\right)$, our Sun will also be screened and the combined effects discussed above are enough to evade the current parameterized post-Newtonian (PPN) constraints.

By assuming that $\phi \rightarrow \phi_{0}$ outside our Galaxy, i.e., that our galactic neighborhood is not screened, these constraints were derived in Hinterbichler \& Khoury (2010) and Brax et al. (2011b) and require

$$
M \lesssim 10^{-3} M_{\mathrm{pl}}
$$

If the assumption about the value of $\phi$ outside our Galaxy, which is very likely to be true, can be relaxed, then the bound above can be relaxed somewhat as well. The constraint on $M$ turns into a constraint on the range of the field and the redshift in which the SSB takes place:

$$
\lambda_{0} \lesssim 2.3 \sqrt{\frac{0.3}{\Omega_{m}}}\left(1+z_{\mathrm{SSB}}\right)^{-3 / 2} \mathrm{Mpc} h^{-1}
$$

Thus, for transitions that take place close to the present time, the fifth force can have a range of at most a few $\mathrm{Mpc} h^{-1}$.

In the rest of this article, instead of working with the parameters $\{\mu, M, \lambda\}$, we will instead choose to work with the more physically intuitive quantities $\left\{L, \beta, z_{\mathrm{SSB}}\right\}$ : the cosmological range of the fifth force in $\mathrm{Mpc} h^{-1}$, the strength of the fifth force relative to gravity, and the redshift at which the SSB takes place in the cosmological background, respectively.

The transformation between the two sets of parameters is given by

$$
\begin{gathered}
\frac{\mu}{H_{0}}=\frac{2998}{\sqrt{2} L} \\
\frac{M}{M_{\mathrm{pl}}}=10^{-3} \sqrt{\frac{\Omega_{m}}{0.27}}\left(\frac{L}{2.36}\right)\left(1+z_{\mathrm{SSB}}\right)^{3 / 2} \\
\lambda=\left(\frac{10^{60} H_{0}}{M_{\mathrm{pl}}} \cdot \frac{0.27}{\Omega_{m}}\right)^{2} \frac{1.38 \times 10^{-100}}{\beta^{2} L^{6}\left(1+z_{\mathrm{SSB}}\right)^{6}} .
\end{gathered}
$$

For typical parameters $L \sim \beta \sim 1$ and $z_{\mathrm{SSB}} \sim 0$ we have $\mu \sim 10^{3} H_{0}, M \sim 10^{-3} M_{\mathrm{pl}}$, and $\lambda \sim 10^{-100}$. Thus, the symmetron is very weakly self-coupled. As with other models with screening mechanisms, our parameters require a certain degree of fine-tuning. This means that SSB is a rather late-time phenomenon.

We will choose to work with values of the parameters that are close to the local constraints and in which the symmetron can produce observable cosmological effects. This means we will be most interested in the parameter space $L=\mathcal{O}(1), \beta=\mathcal{O}(1)$, and $0 \lesssim z_{\text {SSB }} \lesssim 2$. 


\section{SYMMETRON COSMOLOGY}

In this section we discuss the cosmological evolution of the symmetron field from the background evolution to linear perturbations and derive the nonrelativistic limits of the field equations to be implemented in the $\mathrm{N}$-body code. The analysis in this section is mainly for comparison with the $N$-body simulations. For a more thorough discussion regarding the background cosmology and linear perturbations in the symmetron, see Hinterbichler et al. (2011) and Brax et al. (2011b), respectively.

\subsection{Background Cosmology}

The background evolution of the symmetron in a flat Friedmann-Lemaitre-Robertson-Walker (FLRW) metric

$$
d s^{2}=-d t^{2}+a^{2}(t)\left(d x^{2}+d y^{2}+d z^{2}\right)
$$

is determined by the field equation

$$
\ddot{\phi}+3 H \dot{\phi}+V_{\text {eff }, \phi}=0
$$

together with the Friedman equations

$$
\begin{gathered}
3 H^{2} M_{\mathrm{pl}}^{2}=\rho_{m} A(\phi)+\rho_{\phi} \\
\dot{\rho}_{m}+3 H \rho_{m}=0
\end{gathered}
$$

where

$$
\rho_{\phi}=\Lambda-\frac{1}{2} \mu^{2} \phi^{2}+\lambda \phi^{4}+\frac{1}{2} \dot{\phi}^{2} .
$$

When the field follows the minimum of the effective potential, we have

$$
\begin{aligned}
\left|\frac{\rho_{\phi}-\Lambda}{\Lambda}\right| & \lesssim \frac{\mu^{4}}{\lambda \Lambda}=\beta^{2} \frac{\rho_{\mathrm{SSB}}}{\Lambda}\left(\frac{M}{M_{\mathrm{pl}}}\right)^{2} \\
& \lesssim 10^{-6} \beta^{2}\left(1+z_{\mathrm{SSB}}\right)^{3} .
\end{aligned}
$$

Thus, for $\beta, z_{\mathrm{SSB}} \sim \mathcal{O}(1)$ the dynamical part of the potential is too small to contribute significantly to the energy density of the universe and we are left with the cosmological constant to account for dark energy.

In the same regime, the coupling function $A(\phi)$ satisfies

$$
|A(\phi)-1|=\frac{1}{2}\left(\frac{\phi}{M}\right)^{2} \lesssim \beta^{2}\left(\frac{M}{M_{\mathrm{pl}}}\right)^{2} \lesssim 10^{-6} \beta^{2}
$$

which is also too small to produce an observable effect on the background expansion. This implies that the symmetron evades Big Bang Nucleosynthesis (BBN) bounds on the variation of masses of the standard model particles (see Section 3.5). It might be possible to make the symmetron responsible for dark energy by changing the form of the potential and coupling. One such modification was proposed in Hinterbichler et al. (2011); however, it was shown that additional fine-tuning of the parameters was required to yield the desired late-time cosmology.

In Figure 1 we see the background evolution $\phi(z)$ for $z_{\text {SSB }}=2$ together with the analytical minimum. Note that the field does not start to follow the minimum immediately after SSB. This has important consequences for the evolution of the perturbations, which will be discussed in Section 3.4.

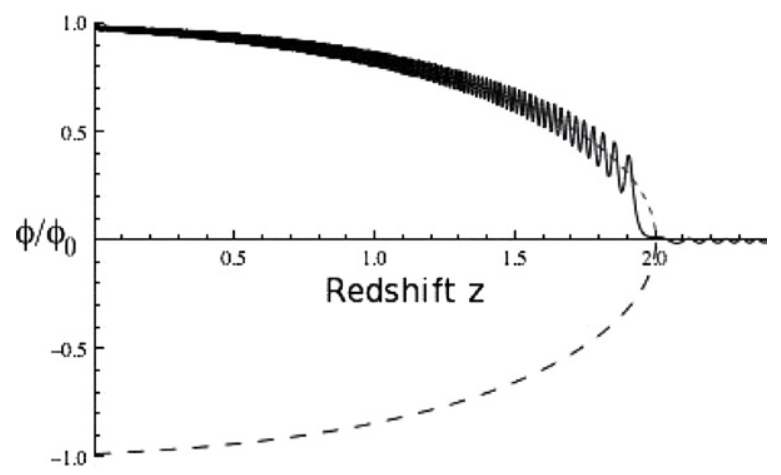

Figure 1. Background evolution of the symmetron for $\beta=1, L=1$, and $z_{\mathrm{SSB}}=2$ together with the analytical background (dashed lines). The symmetry is broken at $z=2$ and the field settles at one of the two branches.

\subsection{Linear Perturbations}

The most general metric in a perturbed FLRW space-time is given by

$$
\begin{aligned}
d s^{2}= & -(1+2 \alpha) d t^{2}-2 a B_{, i} d t d x^{i} \\
& +a^{2}\left((1+2 \psi) \delta_{i j}+2 \gamma_{, i ; j}\right) d x^{i} d x^{j},
\end{aligned}
$$

where the covariant derivative is given in terms of the spatial metric, which in the case of our flat background reduces to $\delta_{i j}$. We decompose the field $\phi$ into the background and perturbated parts: $\phi(\mathbf{x}, t)=\bar{\phi}(t)+\delta \phi(\mathbf{x}, t)$. The energy-momentum tensor of nonrelativistic matter can be decomposed as

$$
T_{0}^{0}=-\rho_{m}\left(1+\delta_{m}\right), T_{i}^{0}=-\rho_{m} v_{i},
$$

where $v$ is the peculiar velocity of nonrelativistic matter and $\delta_{m}$ is the matter density perturbation defined by

$$
\delta_{m} \equiv \frac{\delta \rho_{m}}{\rho_{m}}-\frac{\dot{\rho}_{m}}{\rho_{m}} v \equiv \frac{\delta \rho_{m}}{\rho_{m}} \text { in the comoving gauge }
$$

The equation determining the evolution of the perturbations, neglecting anisotropic stresses, follows from the Einstein equations. The scalar perturbations can be read off from the formulation of Hwang \& Noh (2002), which is independent of gauge. In the following we use units of $M_{\mathrm{pl}} \equiv 1$. After solving for the different metric potentials, we find that the scalar perturbations, in the comoving gauge $(v=0)$, are determined by

$$
\begin{aligned}
\ddot{\delta}_{m} & +2 H \dot{\delta}_{m}-\frac{1}{2} \rho_{m} \delta_{m} \\
& -\frac{\phi \delta \phi}{M^{2}}\left(6 H^{2}+6 \dot{H}+\Omega_{m} H^{2}-\frac{k^{2}}{a^{2}}+2 \dot{\phi}^{2}\right) \\
& -\frac{\phi}{M^{2}}(\ddot{\delta \phi}+5 H \dot{\delta \phi})-\frac{2 \dot{\phi}}{M^{2}}(\dot{\delta \phi}+H \delta \phi) \\
& +V_{\mathrm{eff}, \phi}\left(1+\frac{1}{M^{2}}\right) \delta \phi-2 \dot{\phi} \dot{\delta \phi}=0 \\
\ddot{\delta \phi} & +\left(3 H+\frac{2 \phi \dot{\phi}}{M^{2}}\right) \dot{\delta \phi}+\frac{\phi \rho_{m} \delta_{m}}{M^{2}}-\dot{\phi} \dot{\delta}_{m} \\
& +\left(m_{\phi}^{2}+\frac{k^{2}}{a^{2}}-\frac{2 \phi}{M^{2}} V_{\mathrm{eff}, \phi}+\frac{2 \dot{\phi}^{2}}{M^{2}}\right) \delta \phi=0 .
\end{aligned}
$$



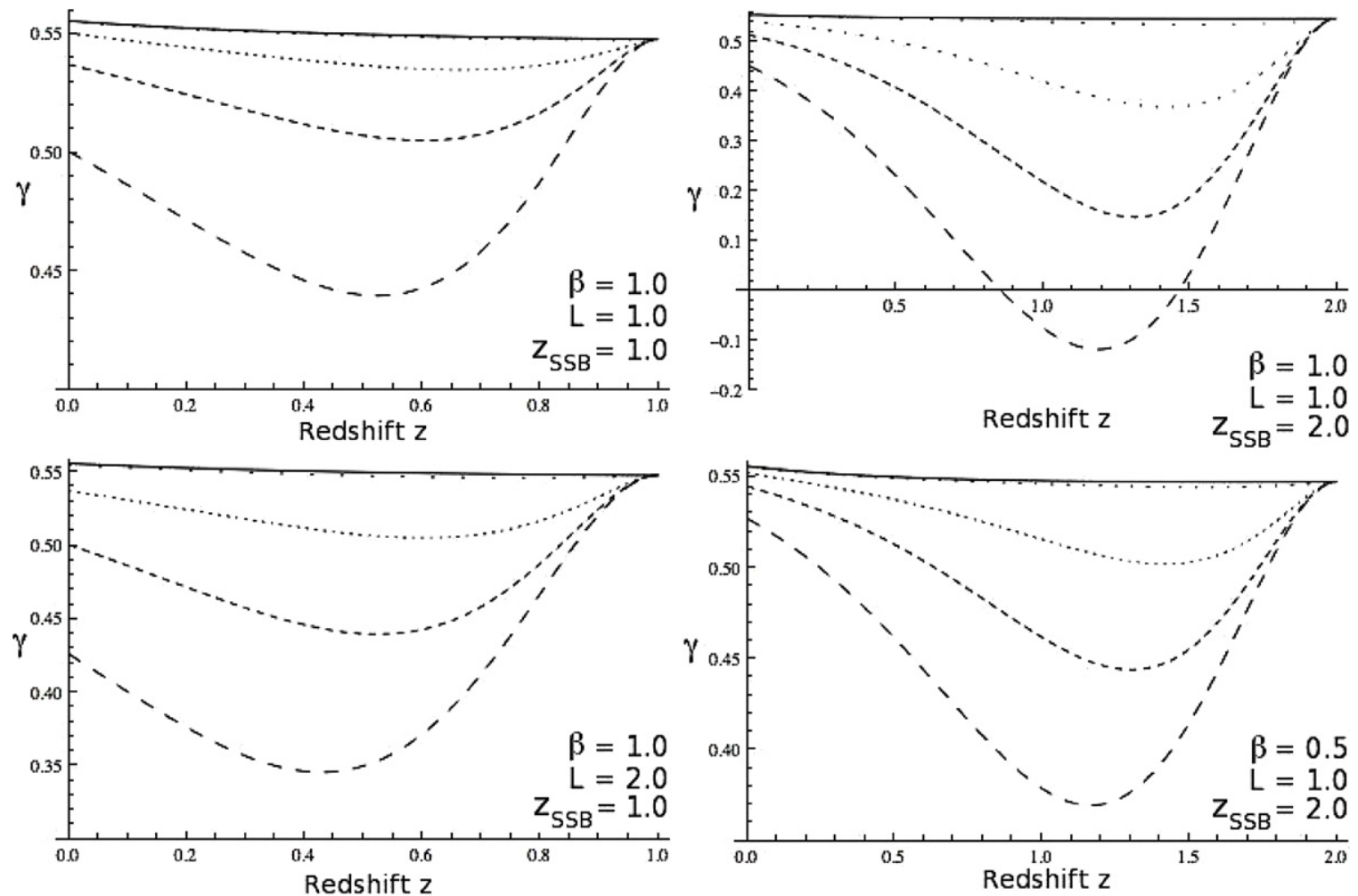

Figure 2. Evolution of the growth index $\gamma(z)$ when $z_{\mathrm{SSB}}=1$ (left) and $z_{\mathrm{SSB}}=2$ (right) for four different wavenumbers $k=\{0.01,0.05,0.1,0.2\} \mathrm{Mpc} h^{-1}$ (from top to bottom in each figure). The solid line shows the prediction of $\Lambda \mathrm{CDM}$.

In studying the perturbations it is convenient to introduce the growth index

$$
\gamma(z, k)=\frac{\log \left(\frac{d \log \delta_{m}}{d \log a}\right)}{\log \left(\Omega_{m}(z)\right)} .
$$

In $\Lambda$ CDM we have $\gamma \approx 0.55$ (for $0.2 \lesssim \Omega_{m} \lesssim 0.3$ ), which is scale and almost redshift independent. In modified theories, however, $\gamma$ can have significant scale and redshift dependence as shown in Gannouji et al. (2010) and Mota \& Winther (2011) for the case of chameleon models, Tsujikawa et al. (2009), Brax et al. (2008, 2010b), Motohashi et al. (2010), Narikawa \& Yamamoto (2010), and Appleby \& Weller (2010) for $f(R)$ modified gravity, and Baldi (2011) for interacting dark energy.

If we assume that the field is rolling slowly along the minimum, we can neglect all terms proportional to $\dot{\phi}$ and the oscillating term $V_{\text {eff, } \phi}$. The perturbations in $\phi$ will evolve more slowly than the perturbations in $\delta_{m}$ for scales deep inside the Hubble radius. Thus, the terms $\rho_{m} \beta,{ }_{\phi} \delta_{m}$ and $\left(m_{\phi}^{2}+\left(k^{2} / a^{2}\right)\right) \delta \phi$ will dominate over the $\delta \phi$ time derivatives in Equation (37). Under these assumptions, we can simplify Equation (36) to

$$
\begin{gathered}
\ddot{\delta}_{m}+2 H \dot{\delta}_{m}=\frac{3}{2} \Omega_{m} H^{2} \frac{G_{\text {eff }}}{G} \delta_{m} \\
\frac{G_{\text {eff }}}{G}=1+\frac{2 \beta^{2} \phi^{2} / \phi_{0}^{2}}{1+\frac{a^{2}}{\lambda_{\phi}^{2} k^{2}}}
\end{gathered}
$$

which are the equations we use to integrate the perturbations.

At times before SSB we have $\phi \approx 0$ and therefore $G_{\text {eff }} \approx G$. After SSB the field approaches the minimum $\phi=\phi_{0}$, in this regime we have

$$
\frac{G_{\text {eff }}}{G}= \begin{cases}1 & \frac{a}{k} \gg \lambda_{\phi} \\ 1+2 \beta^{2} & \frac{a}{k} \ll \lambda_{\phi} .\end{cases}
$$

Thus, small scales will feel a stronger gravitational constant.

In Figure 2 we show the redshift evolution of $\gamma$ for several different wavenumbers, and in Figure 3 we show contour plots for $\gamma(k, z=0)$ for two comoving wavenumbers. The evolution of the growth index is very similar, with a minimum at some redshift $z>0$, to the behavior found in other coupled scalar field models in the literature (see the references below Equation (38)).

The growth rate on really large scales $\left(k \lesssim 0.01 h \mathrm{Mpc}^{-1}\right)$ is not affected by the symmetron fifth force unless $L, \beta \gg 1$. However, on the smallest, linear scales we can still have a deviation from the predictions of GR. Note that we have integrated the perturbations using the approximation Equation (39) instead of the full equations (36) and (37). The explanation for this is given in Section 3.4.

\subsection{Linear Power Spectrum and the CMB}

In Figure 4 we show the factional difference of the linear power spectrum of the symmetron to that of $\Lambda \mathrm{CDM}$, defined as $\Delta P(k) / P(k) \equiv\left(P(k)-P_{\Lambda \mathrm{CDM}}(k)\right) / P_{\Lambda \mathrm{CDM}}(k)$. Note that on linear scales $\left(k \lesssim 0.1 h \mathrm{Mpc}^{-1}\right)$ the power spectrum is very close to $\Lambda$ CDM. Going down to scales comparable to the length scale of the symmetron $\left(k \sim L^{-1} h \mathrm{Mpc}^{-1}\right)$ the power spectrum starts to deviate significantly. However, in this regime the perturbations are already nonlinear and we cannot trust the results of the linear perturbation theory. Once we discuss the $N$-body results, we will see that the symmetron mechanism is at work in this regime, thereby suppressing the fractional difference from $\Lambda \mathrm{CDM}$ in the power spectrum predicted by linear perturbation theory. 

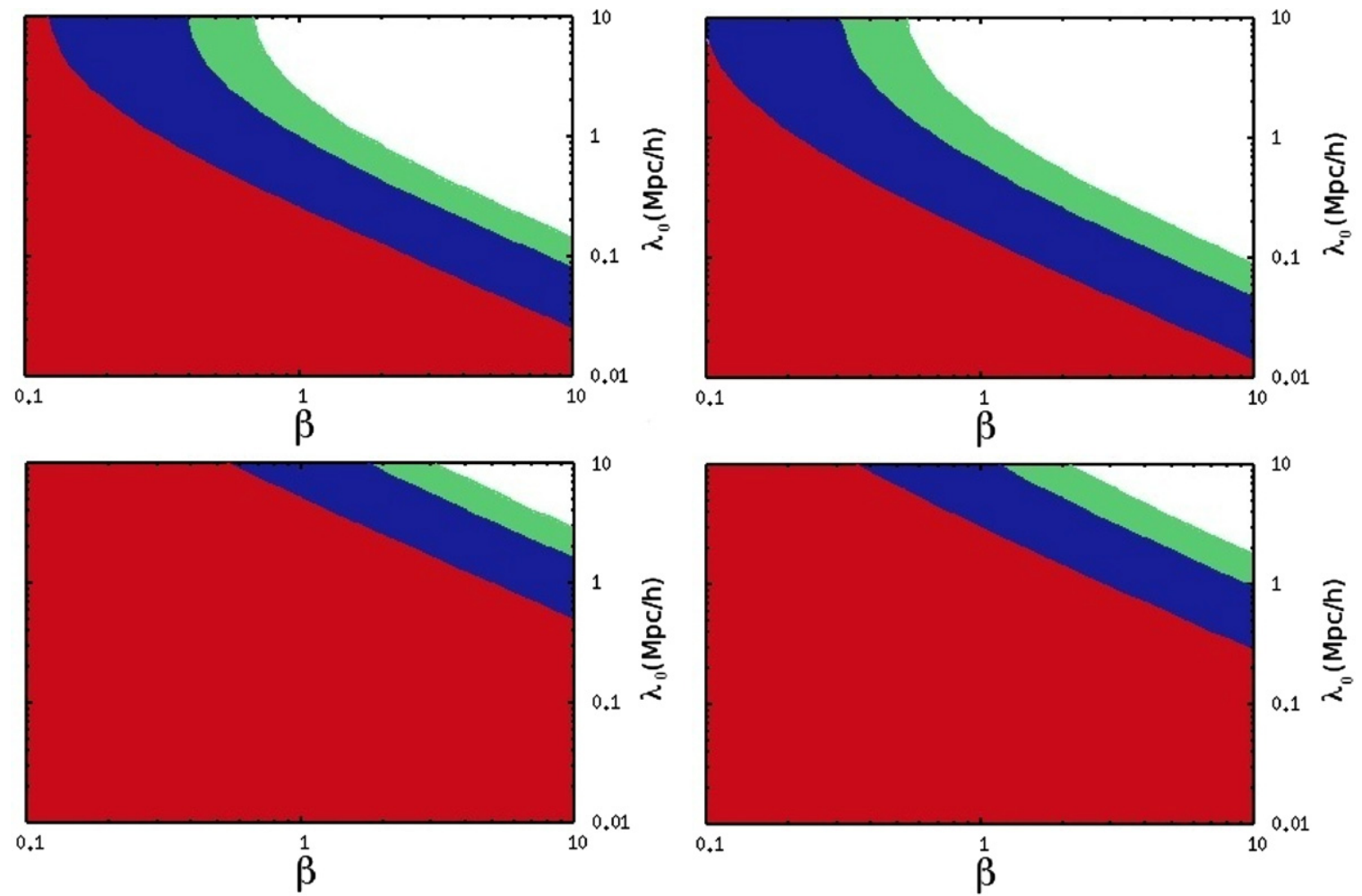

Figure 3. Growth index $\gamma(z=0)$ for $z_{\mathrm{SSB}}=1.0$ (left) and $z_{\mathrm{SSB}}=2.0$ (right) for two comoving wavenumbers: $k=0.2 \mathrm{Mpc} h^{-1}$ (above) and $k=0.01 \mathrm{Mpc} h^{-1}$ (below). The red region shows the GR regime $\gamma \simeq 0.555$, the blue region shows the regime where $0.5<\gamma<0.55$, the green region shows $0.4<\gamma<0.5$, and the white region shows $\gamma<0.4$.

(A color version of this figure is available in the online journal.)

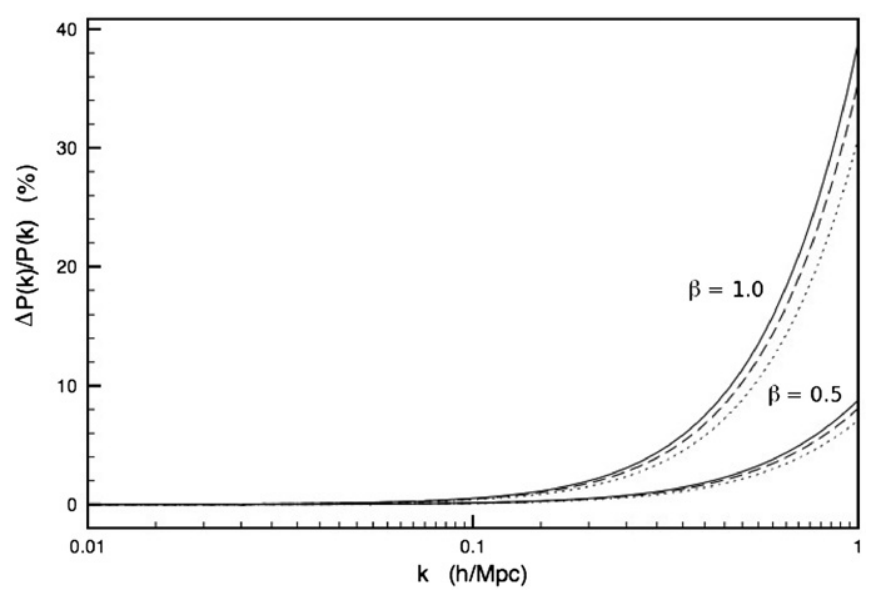

Figure 4. Linear power spectrum relative to that of $\Lambda \mathrm{CDM}$ for three different $\mathrm{SSB}$ redshifts: $z_{\mathrm{SSB}}=0.5$ (dotted), $z_{\mathrm{SSB}}=1.0$ (dashed), and $z_{\mathrm{SSB}}=2.0$ (solid). We have fixed $L=1$ and shown the results for the two values $\beta=0.5$ and $\beta=1.0$.

The short range $(\lesssim \mathrm{Mpc})$ of the fifth force means that it will not affect the cosmic microwave background (CMB) unless $L, \beta \gg$ 1. Take $L=1$ and $\beta=2$ as an example: we find a maximal increase in power (due to the integrated Sacks-Wolfe effect) of $\sim 0.25 \%$ for multipoles around $l \sim 100$. One needs a much larger $\beta$ and/or $L$ to have a detectable signature in the CMB. The second case is not allowed by local experiments, while the first case implies a growth rate of the linear perturbations that should have difficulty satisfying constraints coming from large-scale structure surveys.
A more thorough analysis of the linear perturbations in the symmetron model can be found in Brax et al. (2011b). There it was shown that strong signatures appear in other interesting linear observables such as the weak-lensing slip parameter and the modified gravity parameter.

\subsection{Tachyonic Instability in the Perturbations}

The perturbations in Section 3.2 were integrated using the approximate Equation (39), which is equivalent to using the analytical minimum as the background field. The reason we did not use the full equations is because perturbation theory breaks down close to $z_{\mathrm{SSB}}$.

Immediately before $z_{\mathrm{SSB}}$ the field is still close to $\phi=0$; as $z \rightarrow z_{\text {SSB }}$ the mass of the field vanishes. This means that the field cannot follow the minimum and starts to lag behind as seen in Figure 1. The global minimum of the effective potential $\phi=0$ now becomes a local maximum, and the mass squared of the field becomes negative, leading to an exponential growth in the perturbations.

To see what happens, we can simplify Equation (37) by discarding all but the most important terms:

$$
\ddot{\delta \phi}+\left(m_{\phi}^{2}+\frac{k^{2}}{a^{2}}\right) \delta \phi \simeq 0 .
$$

If $m_{\phi}^{2}+k^{2} / a^{2}<0$, then the solution to the above equation reads $\delta \phi \propto \exp \left(t \sqrt{\left|m_{\phi}^{2}+k^{2} / a^{2}\right|}\right)$, which is exponentially growing.

In a realistic situation the field would roll very quickly down from the false minimum $\phi=0$, making $m_{\phi}^{2}$ positive and thereby stabilizing the field close to the symmetry breaking minimum (Felder et al. 2001). Since perturbation theory is only valid as 
long as the perturbations $\delta \phi$ are small, we get a breakdown of the perturbation theory when using the true background solution. The blowup in $\delta \phi$, in turn, leads to a blowup in the matter perturbations, and the numerical results cannot be trusted.

We have investigated this further by using $N$-body simulations. In Figure 10 we see a snapshot of the $\phi$-distribution both before and after $z=z_{\mathrm{SSB}}=2.0$. There we see the same sort of behavior as is familiar from symmetry breaking in condensed matter physics: symmetry breaking takes place at different places at different times according to the local matter density. This type of dynamics is not taken care of in the standard perturbation theory approach, which leads to the apparent instability.

Note that by using the analytical minimum when integrating the perturbations we do not have control over the accuracy of our results. A full analysis of this phenomenon could be handled with $N$-body simulations, but in our simulations we have not explicitly taken into account the time variation of the scalar field (we work in the quasi-static limit), and our simulation box is also too small to reach far enough into the linear regime. We leave this study for future work.

\subsection{Varying Constants}

One important constraint on coupled scalar field theories comes from the time variation of the gravitational constant $G$ in the Jordan frame, or equivalently in the masses of the standard model particles in the Einstein frame. Wilkinson Microwave Anisotropy Probe (WMAP) constrains any such variation to be less than about 5\% since recombination (Nagata et al. 2004). Light-element abundances provide similar constraints between the time of nucleosynthesis and today (Accetta et al. 1990).

Due to the conformal coupling to matter, $A(\phi)$, a constant mass scale in the Jordan frame becomes time and space varying in the Einstein frame. The mass variation between today and recombination is given by

$$
\frac{\Delta m}{m}=\frac{A\left(\phi_{\mathrm{rec}}\right)-A\left(\phi_{\text {today }}\right)}{A\left(\phi_{\mathrm{rec}}\right)} \simeq \frac{1}{2}\left(\frac{\phi_{\text {today }}}{M}\right)^{2},
$$

where we have put $\phi_{\text {rec }} \simeq 0$ since $z_{\text {rec }} \gg z_{\text {SSB }}$ in all interesting cases. If we further assume $\phi_{\text {today }}=\phi_{0}$, we get the conservative constraint

$$
\frac{\Delta m}{m} \simeq \frac{1}{2}\left(\frac{\phi_{0}}{M}\right)^{2}=\beta^{2}\left(\frac{M}{M_{\mathrm{pl}}}\right)^{2}<10^{-6} \beta^{2} .
$$

The WMAP constraint $|\Delta m / m| \lesssim 0.05$ is satisfied for all $\beta \lesssim 100$.

Analysis of absorption spectra of quasars has led some to claim that the fine structure constant $\alpha$ might have evolved by approximately one part in $10^{5}$ over the redshift range $0.2<z<3.7$. If this turns out to be true, then general covariance would imply that $\alpha$ can vary both in space and in time, that is, it must be a function of a field.

Since we have so far assumed that the symmetron couples conformally to matter fields, and since the Maxwell action is conformally invariant, at tree level the symmetron does not lead to a time-varying $\alpha$. By considering a coupling of the symmetron to photons of the form

$$
S_{\gamma}=-\frac{1}{4} \int d^{4} x \sqrt{-g} A_{\gamma}(\phi) F_{\mu \nu} F^{\mu \nu}
$$

where

$$
A_{\gamma}(\phi)=1+\frac{\zeta_{\gamma}}{2}\left(\frac{\phi}{M}\right)^{2}
$$

then variations in $\phi$ will lead to variations in $\alpha$. Here $\zeta_{\gamma}$ is the symmetron-photon coupling relative to the symmetron-matter coupling. The variation in the fine-structure constant between Earth $(E)$ and another place $(S)$ in the universe is given by

$$
\left|\frac{\Delta \alpha}{\alpha}\right|=\frac{A_{\gamma}\left(\phi_{E}\right)-A_{\gamma}\left(\phi_{S}\right)}{A_{\gamma}\left(\phi_{E}\right)} \simeq \frac{\zeta_{\gamma}}{2}\left(\frac{\phi_{S}}{M}\right)^{2} .
$$

If $S$ is a very low density environment where $\phi_{S} \approx \phi_{0}$, then

$$
\left|\frac{\Delta \alpha}{\alpha}\right| \simeq \zeta_{\gamma} 10^{-6} \beta^{2}
$$

which for $\mathcal{O}(1) \lesssim \beta, \zeta_{\gamma}$ is close to the reported detection.

However, the local density in most Ly $\alpha$-emitting systems is usually much larger than the cosmological background density today (see, e.g., Brax et al. 2004 and references therein), which implies $\phi_{S} \ll \phi_{0}$ and the above estimate becomes even smaller.

To be able to account for the reported claims, we need $z_{\mathrm{SSB}}$ to be well before the observed redshift of these systems and/or these systems to be located in voids to produce the desired $10^{-5}$ effect. This makes it possible that the symmetron is responsible for the claimed variations, but will probably require a fine-tuning $\zeta_{\gamma} \gg 1$. A more detailed analysis, as done in Li et al. (2011b), is required to see if this is the case. This is beyond the scope of this paper.

\subsection{N-body Equations}

To implement the general relativistic Equations (5), (12), (13), and (16) in $N$-body simulations, it suffices to work in the nonrelativistic limits, since the simulations only probe the weak gravity regime and small volumes compared with the cosmos. We write the perturbed metric in the (flat) conformal Newtonian gauge as

$$
d s^{2}=-a^{2}(1+2 \Xi) d \tau^{2}+a^{2}(1-2 \Psi) d x^{\mu} d x_{\mu},
$$

where $\tau$ is the conformal time and $x^{\mu}$ is the comoving coordinate. In Appendix A we list the expressions for the Christoffel symbols, the Ricci tensor, and the Ricci scalar for the metric Equation (49), which are used in deriving the equations below.

The scalar field equation of motion in terms of the perturbed quantities becomes

$$
\begin{aligned}
& -(1-2 \Xi) \phi^{\prime \prime}+\nabla_{\mathbf{x}}^{2} \phi-\phi^{\prime}\left(2 H(1-2 \Xi)-\Xi^{\prime}-3 \Psi^{\prime}\right) \\
& =a^{2}\left(\phi\left(\frac{\rho_{m}}{M^{2}}-\mu^{2}\right)+\lambda \phi^{3}\right) .
\end{aligned}
$$

Taking the quasi-static limit of this equation, in which we can neglect terms such as $\Xi^{\prime}, \Psi^{\prime}$, and $H \phi^{\prime}$ since the time derivative of a quantity is much smaller than its spatial gradient, and removing the background part, we obtain

$$
\begin{aligned}
\nabla_{\mathbf{x}}^{2} \phi & \approx \frac{a^{2}}{M^{2}}\left(\rho_{m} \phi-\bar{\rho}_{m} \bar{\phi}\right) \\
& +a^{2}\left(\mu^{2}(\bar{\phi}-\phi)+\lambda\left(\phi^{3}-\bar{\phi}^{3}\right)\right)
\end{aligned}
$$

where we have also used the approximation $A(\phi) \approx 1$ to simplify the equation further. 
The $(0,0)$-component of the Ricci tensor and the trace of the total energy-momentum tensor in the perturbed quantities become

$$
\begin{aligned}
& a^{2} R_{0}^{0} \approx-\nabla_{\mathbf{x}}^{2} \Xi+3\left(\frac{a^{\prime \prime}}{a}-H^{2}\right)(1-2 \Xi) \\
&-3 \Psi^{\prime \prime}-3 H\left(\Xi^{\prime}+\Psi^{\prime}\right) \\
& T \approx-A(\phi) \rho_{m}-4 V(\phi)+\frac{1}{a^{2}}(1-2 \Psi) \phi^{\prime 2} .
\end{aligned}
$$

The $(0,0)$-component of the Einstein equation with the background part removed gives the nonrelativistic Poisson equation

$$
\nabla_{\mathbf{x}}^{2} \Phi \approx 4 \pi G\left(\rho_{m}-\bar{\rho}_{m}\right) a^{3}
$$

where we have neglected the contribution from the potential $(V(\phi)-V(\bar{\phi}))$, put $A(\phi) \approx 1$, and taken $\Phi=a \Xi$ for convenience.

The equation of motion for the $N$-body particles follows from the geodesic equation and reads

$$
\ddot{\mathbf{x}}+2 H \dot{\mathbf{x}}=-\frac{1}{a^{3}} \nabla_{\mathbf{x}} \Phi-\frac{1}{a^{2}} \frac{\phi}{M^{2}} \nabla_{\mathbf{x}} \phi-\frac{\phi \dot{\phi}}{M^{2}} \dot{\mathbf{x}} .
$$

By rewriting this equation in terms of the conjugate momentum to $\mathbf{x}, \mathbf{p}=a^{2} \mathbf{x}$, we have

$$
\begin{gathered}
\frac{d \mathbf{x}}{d t}=\frac{\mathbf{p}}{a^{2}} \\
\frac{d \mathbf{p}}{d t}=-\frac{1}{a} \nabla_{\mathbf{x}} \Phi-\frac{\phi}{M^{2}}\left(\nabla_{\mathbf{x}} \phi+\dot{\phi} \mathbf{p}\right) .
\end{gathered}
$$

Equations (51), (54), and (56) are all we need to put into the $\mathrm{N}$-body simulation code in order to study structure formation in the nonlinear regime. The discretization of these equations, as implemented in the $\mathrm{N}$-body code, is shown in Appendix B.

\section{4. $N$-BODY SIMULATIONS}

Below we describe the algorithm and model specifications of the $N$-body simulations we have performed. We also give results from tests of the code to show that the scalar field solver works accurately.

\subsection{Outline}

For our simulations we have used a modified version of the publicly available $N$-body code MLAPM (Knebe et al. 2001). The modifications we have made follow the detailed prescription of Li \& Barrow (2011a), and here we only give a brief description. The MLAPM code has two sets of meshes: the first includes a series of increasingly refined regular meshes covering the whole cubic simulation box, with, respectively, 4 , $8,16, \ldots, N_{d}$ cells on each side, where $N_{d}$ is the size of the domain grid, which is the most refined of these regular meshes. This set of meshes is needed to solve the Poisson equation using the multigrid method or fast Fourier transform (for the latter only the domain grid is necessary). When the particle density in a cell exceeds a pre-defined threshold, the cell is further refined into eight equally sized cubic cells; the refinement is done on a cellby-cell basis, and the resulting refinement could have arbitrary
Table 1

The Symmetron Parameters Used in Our Simulations

\begin{tabular}{lccccccccc}
\hline \hline Model & A & B & C & D & E & F & G & H & $\Lambda$ CDM \\
\hline$z_{\text {SSB }}$ & 0.5 & 0.5 & 1.0 & 1.0 & 2.0 & 2.0 & 1.0 & 1.0 & 0.0 \\
$\beta$ & 0.5 & 1.0 & 0.5 & 1.0 & 0.5 & 1.0 & 0.5 & 1.0 & 0.0 \\
$L$ & 1.0 & 1.0 & 1.0 & 1.0 & 1.0 & 1.0 & 2.0 & 2.0 & 0.0 \\
\hline
\end{tabular}

shape, which matches the true equal density contours of the matter distribution. This second set of meshes is used to solve the Poisson equation using the linear Gauss-Seidel relaxation scheme.

The symmetron field is the most important ingredient in the model studied here, and we have to solve for it to obtain detailed information about the fifth force. In our $N$-body code, we have added a new scalar field solver. It uses a nonlinear Gauss-Seidel scheme for the relaxation iteration and the same criterion for convergence as the default Poisson solver in MLAPM. However, it uses $\mathrm{V}$-cycle instead of the self-adaptive scheme in arranging the Gauss-Seidel iterations.

The modified Poisson equation is then solved using nonlinear Gauss-Seidel relaxation on both the domain grid and the refinements. With the gravitational potential $\Phi$ and the scalar field $\phi$ at hand, we can evaluate the total force on the particles and update their momenta (velocities), which are used to advance the particles in space.

\subsection{Simulation Details}

The physical parameters we use in the simulations are as follows: the present dark-energy energy density $\Omega_{\Lambda}=$ 0.733 , total matter density $\Omega_{m}=\Omega_{b}+\Omega_{c}=0.267$, baryon density $\Omega_{b}=0.045$, CDM density $\Omega_{c}=0.222, H_{0}=$ $71.9 \mathrm{~km} \mathrm{~s}^{-1} \mathrm{Mpc}^{-1}, n_{s}=0.963$, and $\sigma_{8}=0.801$. We use a simulation box with size $64 \mathrm{Mpc} h^{-1}$, in which $h=$ $H_{0} /\left(100 \mathrm{~km} \mathrm{~s}^{-1} \mathrm{Mpc}^{-1}\right)$. We simulate nine different models; see Table 1 for the symmetron parameter values.

These parameters are chosen so that they predict local fifth forces that are of the same order of magnitude as allowed by current experiments and observations and are such that we can see the effect of the different parameters. In this way the results from our $N$-body simulations will show the maximum allowed deviation from $\Lambda C D M$. Note that the energy density in the symmetron is always much less than that of dark energy and therefore does not alter the background cosmology, which in all runs will be that of $\Lambda \mathrm{CDM}$.

In all those simulations, the particle number is $256^{3}$, so that the mass resolution is $1.114 \times 10^{9} \mathrm{Mpc} h^{-1}$. The domain grid is a $128 \times 128 \times 128$ cubic and the finest refined grids have 16,384 cells on each side, corresponding to a force resolution of about $12 \mathrm{kpc} h^{-1}$. The force resolution determines the smallest scale on which the numerical results are reliable. Our simulations are purely $\mathrm{N}$-body, and baryons are treated as CDM, which means that no baryonic physics has been included in the numerical code.

The simulation box used, $B=64 \mathrm{Mpc} h^{-1}$, is small compared to linear scales, and we are therefore possibly neglecting effects of mode coupling between linear and nonlinear scales. This will have to be checked by simulations with a larger box size. However, since the fifth force has a short range $(\lesssim \mathrm{Mpc})$, it does not reach far into the linear regime (see Figure 11), and therefore we expect the accuracy on large scales to be the same as for the $\Lambda \mathrm{CDM}$ simulations. 


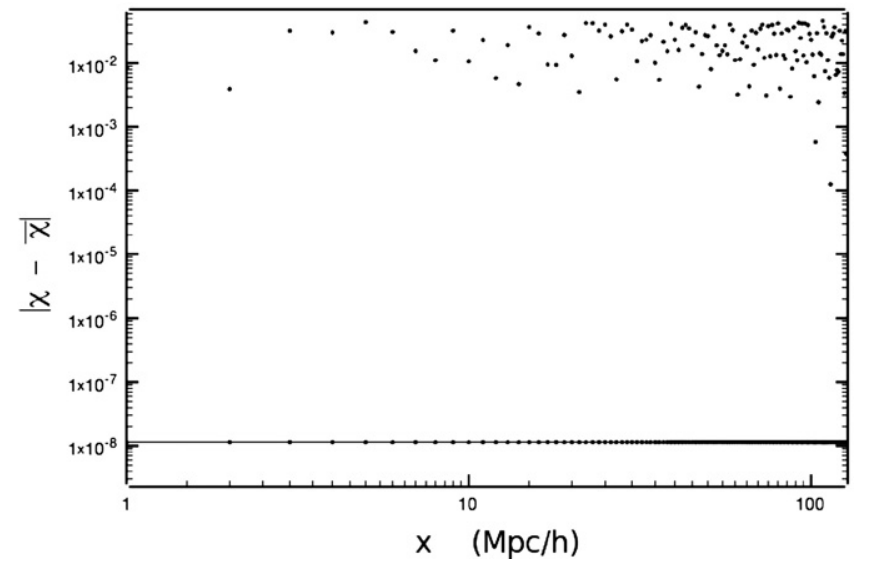

Figure 5. Scalar field relative to the analytical solution before (random initial values above) and after (below) the Newton-Gauss-Seidel relaxation.

\subsection{Initial Conditions}

Initial conditions for the simulation were generated using GRAFIC2 (Bertschinger 2001; Prunet et al. 2008) by using the parameters described above. The same initial conditions were used for all the simulations in order to see clearly the effect of the symmetron compared with $\Lambda$ CDM.

This choice needs some justification. First of all, we start the simulation at $z=49$, a time in which the symmetron has no effect on the growth of the perturbations. This means that the only change the symmetron will have on the initial conditions is on the value of $\sigma_{8}$ today, which is used to normalize the perturbations. Since the symmetron field has a rather short range compared to the linear regime, we do not expect a large effect on $\sigma_{8}$ for the range $L \lesssim \mathcal{O}(1)$ we are considering.

To check this assumption, we integrated the perturbations and calculated the value of $\sigma_{8}$ (by normalizing to the CMB) for our simulation models and found that the model with $L=1$ that is furthest away from $\Lambda \mathrm{CDM}$, namely, $F$ in which $z_{\mathrm{SSB}}=2.0$, $L=1$, and $\beta=1$, only has $\sigma_{8} \simeq 1.01 \sigma_{8}^{\mathrm{LCDM}}$, justifying the use of $\Lambda \mathrm{CDM}$ initial conditions.

If one is to consider models in which $L$ is much larger than 1, then this becomes an issue that should be dealt with properly.

\subsection{Code Tests}

Before we run simulations, we have to make sure that the scalar field solver, which is the main modification to the MLAPM code, works accurately by performing code tests for situations where the outcome is known from analytical solutions.

The scalar field solver uses the nonlinear Newton-GaussSeidel relaxation scheme to compute $\chi \equiv \phi / \phi_{0}$, and an indicator that it works is to show that, given the initial guess of the solution that is very different from the true solution, the relaxation could produce the latter within a reasonable number of iterations. We consider a simulation box with homogeneous density (obtained by putting particles on a regular grid inside the simulation box); then the true solution is given by $\chi=\bar{\chi}$ : the background solution. We therefore make an initial guess for $\chi$ that is randomly scattered around $\bar{\chi}$ and let the scalar field solver solve for $\chi$. The results for $|\chi-\bar{\chi}|$ before and after the relaxation scheme are shown in Figure 5. The difference between the initial guess and the true solution varies between 0.001 and 0.1 , while after the relaxation the difference is of order $10^{-8}$. By using double-precision numbers in all the calculations, we obtained exactly the analytical solution (to double precision

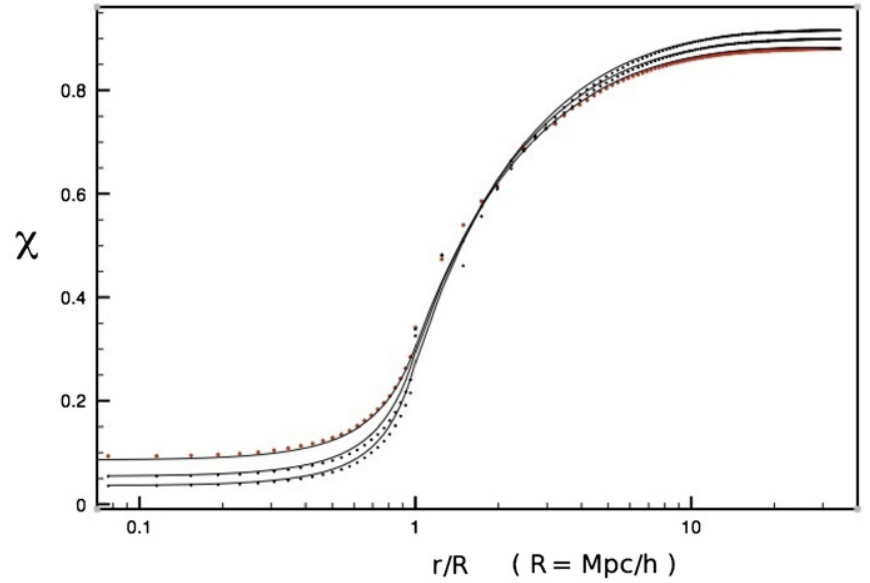

Figure 6. Scalar field value as a function of distance from the center for a spherical overdensity embedded in a background of homogenous density $\rho_{b}$ together with the analytical solution for $\rho_{c}=4000,6000$, and 8000 times $\rho_{b}$. The points shown here are calculated by binning the scalar field value using a bin width $\Delta(r / R)=0.01$ and taking the average. We used the same amount of particles, $128^{3}$, in each run so that the background density $\rho_{b}$ differs for the three cases shown above.

$\approx 10^{-15}$ ), while using only floating point numbers the accuracy dropped to $10^{-6}$, which is exactly the accuracy in floats. This shows that the scalar field solver works accurately.

The most important effect of the symmetron is the screening mechanism, in which the local value of the field should be pushed down toward $\chi=0$ in high-density environments. We therefore consider a spherical overdensity, located at the center of the box, with a given radius $R$, homogeneous density $\rho_{c}$ inside $R$, and embedded in a background of homogenous density $\rho_{b}$. The analytical solution reads

$$
\begin{gathered}
\chi(r)=\chi(0) \frac{\sinh \left[m_{c} r\right]}{m_{c} r}, \quad r<R \\
\chi(r)=\chi_{b}+\frac{\left(\chi(R)-\chi_{b}\right) R}{r} e^{-m_{b}(r-R)}, \quad r>R
\end{gathered}
$$

where

$$
\begin{aligned}
m_{c}^{2} & \simeq\left(\frac{\rho_{c}}{M^{2}}\right), \quad m_{b}^{2} \simeq\left(\frac{\rho_{b}}{M^{2}}+\mu^{2}\left(3 \chi_{b}^{2}-1\right)\right) \\
\chi_{b} & \simeq \sqrt{1-\frac{\rho_{b}}{\rho_{\mathrm{SSB}}}}, \quad \chi(R)=\chi_{b}\left(\frac{1+m_{b} R}{\frac{m_{c} R}{\tanh \left(m_{c} R\right)}+m_{b} R}\right) \\
\chi(0) & =\chi(R) \frac{m_{c} R}{\sinh \left(m_{c} R\right)} .
\end{aligned}
$$

For the trial solution on the grid we use the background value $\chi_{b}$ and perform the test for a range of densities $\rho_{c}$. The results after relaxation for the most massive cases are shown in Figure 6. There are some small discrepancies from the analytical solution in the region $R<r<2 R$ for the most extreme cases $\rho_{c}>10^{3} \rho_{b}$. This is not a surprise as the density suddenly drops over 3 orders of magnitude at $r=R$, meaning that we need a lot of particles in this region in order to get accurate results. In the region $r<R$ and $r>2 R$ the scalar field solver produces the analytical solution to high accuracy.

\section{NUMERICAL RESULTS}

In this section we present the results from the simulations, including the snapshots, the matter power spectrum, and the halo mass function. 

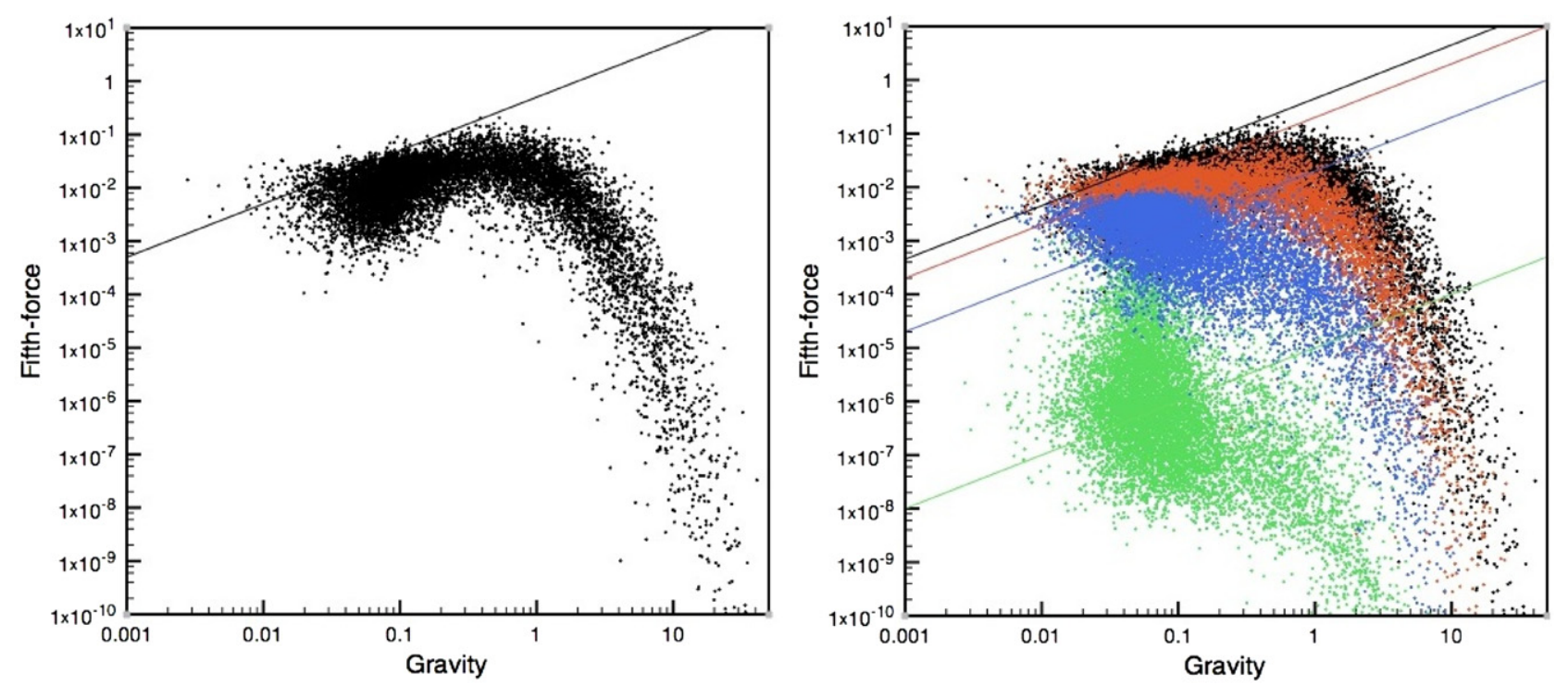

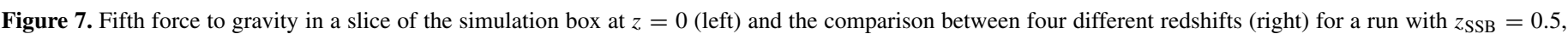

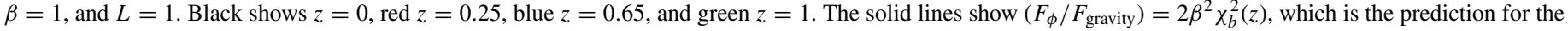

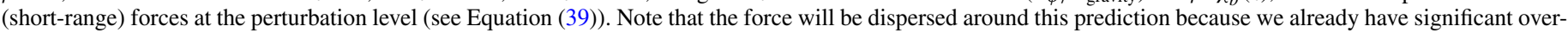

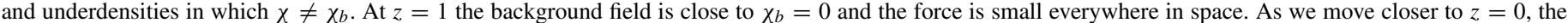

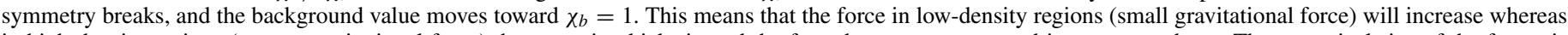

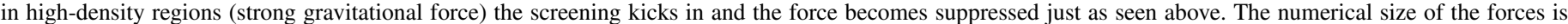
given in terms of code units, which are $H_{0}^{2} / B$ times the physical force unit.

(A color version of this figure is available in the online journal.)

\subsection{Snapshots}

In the symmetron model $\chi \equiv \phi / \phi_{0}$, and thereby the fifth force, is suppressed in high-density regions. In this subsection we demonstrate these qualitative features using some snapshots.

Figure 7 shows the ratio of the fifth force to gravity today and for redshifts both before and after $z_{\mathrm{SSB}}$.

At early times, the density is high everywhere and we expect the fifth force on all particles to be strongly suppressed. At later times we expect a screening in regions of high matter density. These predictions are confirmed in Figure 7. We see that fifth force on the particles that feel a strong gravitational force (i.e., particles in a high-density environment) is highly suppressed whereas the fifth force on particles that feel a weak gravitational force (i.e., particles in a low-density environment) follows the unscreened theoretical prediction $F_{\phi} \simeq 2 \beta^{2} \chi_{b}^{2}(z) F_{\text {gravity }}$ (see Equation (39)).

Figures $8-10$ show the density and scalar field distribution in a slice of the simulation box at different redshifts for the three cases $z_{\mathrm{SSB}}=0.5,1.0$, and 2.0 with $\beta=L=1.0$ fixed.

For redshifts $z>z_{\mathrm{SSB}}, \chi$ is very close to the minimum $\chi=0$ almost everywhere in space except in voids where the symmetry has already been (weakly) broken. When we go down to redshifts $z<z_{\mathrm{SSB}}$, the symmetry is broken in most parts of the box, except in the high-density regions where we still have $\chi \sim 0$. Comparing the scalar field distribution today for runs with different $z_{\mathrm{SSB}}$, we see that the earlier the symmetry breaking takes place, the part of the box that is unscreened $(\chi \sim 1)$ today becomes larger. This is because the critical density for the symmetry breaking is larger for larger $z_{\mathrm{SSB}}$ and therefore the halos have to be more massive in order to be effectively screened.

\subsection{Matter Power Spectrum}

The nonlinear matter power spectrum is an important observable and could be used to distinguish between different models of structure formation. As we have seen above, the symmetron can have a strong effect on the growth rate of the linear perturbations for parameters that are allowed by local experiments. We expect these signatures to show up in the nonlinear matter power spectrum.

Figure 12 displays the fractional difference in the matter power spectrum from that of $\Lambda \mathrm{CDM}$, defined as $(P(k)-$ $\left.P_{\Lambda \mathrm{CDM}}(k)\right) / P(k)$, and in Figure 11 we show the actual power spectra for the symmetron and $\Lambda \mathrm{CDM}$ together with the corresponding predictions from linear perturbation theory.

Generally, the power spectrum is expected to be reliable up to the scale

$$
k_{\max }=N_{\mathrm{eff}} k_{N / 2},
$$

where $k_{N / 2}=\pi N_{p}^{1 / 3} /(4 B)$ is half the particle Nyquist scale, $N_{p}$ is the total number of particles, $B$ is the box size, and $N_{\text {eff }}$ is a factor determined by the adaptive nature of the code. For nonadaptive simulations $N_{\text {eff }}=1$ and it was shown in Stabenau \& Jain (2006) that the power spectra cannot be trusted for wavenumbers much larger than $k_{N / 2}$.

The MLAPM code, on the other hand, is adaptive, meaning that $N_{\text {eff }}>1$, and allows us to go beyond the half Nyquist scale. The exact value of $N_{\text {eff }}$ depends on the number of refinements triggered in the whole simulation process, and for our simulations we estimate $N_{\text {eff }}=8-10$. To be conservative, we follow Zhao et al. (2011), which uses the same simulation details as us but for the case of $f(R)$ gravity, and take $N_{\text {eff }}=7$. This translates into an estimate $k_{\max } \simeq 22 \mathrm{~h} \mathrm{Mpc}^{-1}$ for the validity of our results.

This estimate can be invalidated by the contribution from shot noise due to limited resolution at small scales. In Figure 13 we show the expected shot-noise contribution in one of our simulations together with the statistical error in the power spectrum estimation computed by POWMES. We define $k_{\mathrm{SN}}$ to be the wavenumber such that the expected shot-noise contribution is 

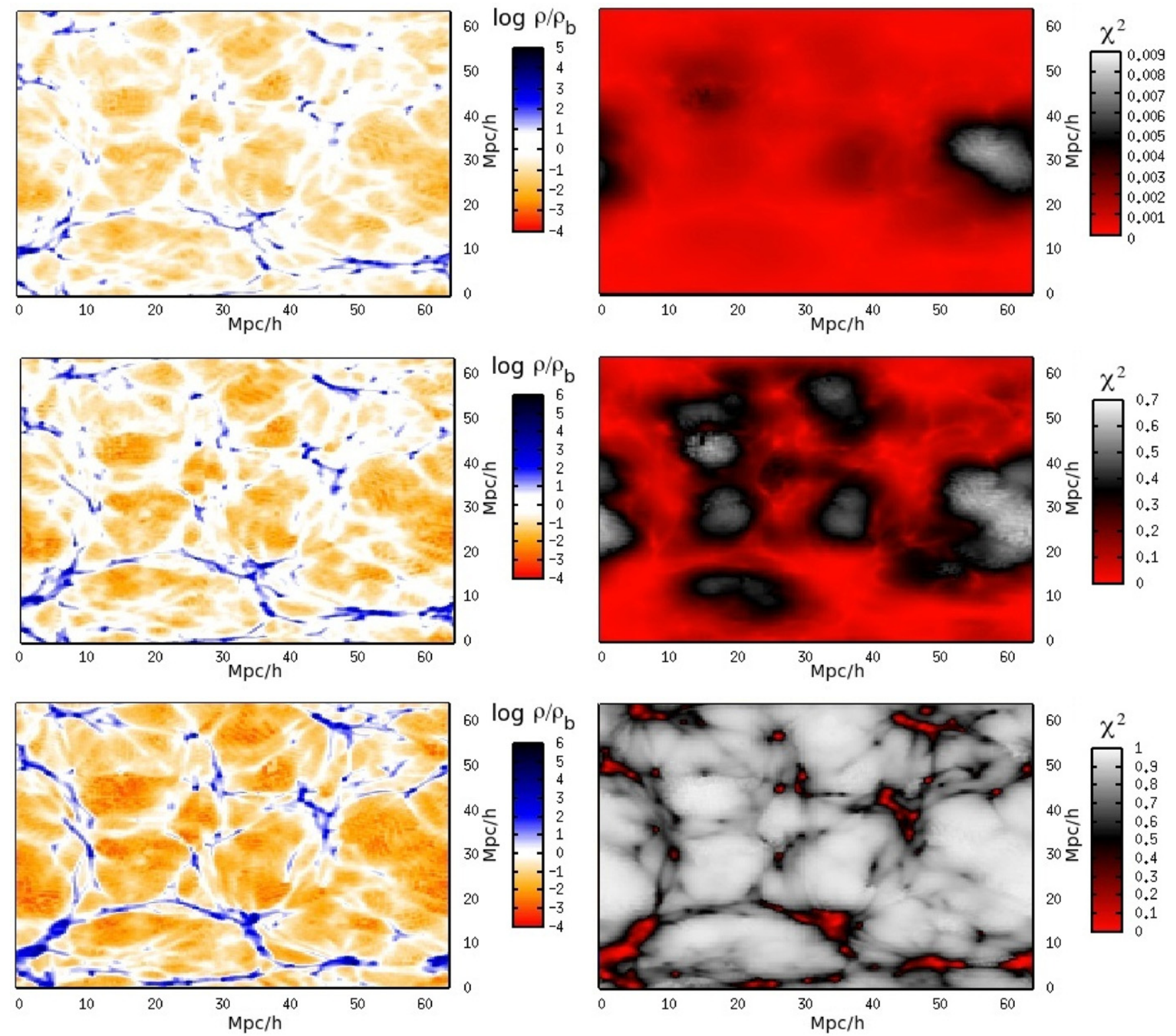

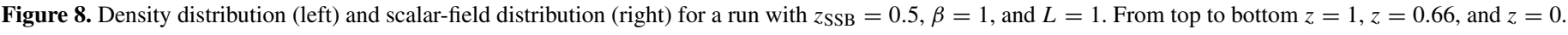
(A color version of this figure is available in the online journal.)

below $5 \%$ for all $k<k_{\mathrm{SN}}$ up to $z=z_{\mathrm{SSB}}$, which is the redshift for which the symmetron power spectrum starts deviating from $\Lambda \mathrm{CDM}$, and overplot the scale $k=\min \left(k_{\max }, k_{\mathrm{SN}}\right)$ in Figures 12 and 14. For all of our simulations we find $k_{\mathrm{SN}} \gtrsim k_{\max }$ except when $z_{\mathrm{SSB}}=2.0$, where $k_{\mathrm{SN}} \simeq 12 \mathrm{~h} \mathrm{Mpc}^{-1}$.

The power spectrum agrees with the predictions of linear perturbation theory on large scales $\left(k \lesssim 0.1 \mathrm{Mpc}^{-1}\right)$, but on smaller scales the results found here are weaker than the prediction of linear perturbation theory seen in Figure 4. This is because when linearizing the field equation we are basically using the background matter density everywhere and therefore preventing the symmetron mechanism from taking effect in suppressing the fifth force when matter perturbations become large. In contrast, the $N$-body simulation avoids this approximation by taking full account of the suppression of the fifth force.

The fractional difference relative to $\Lambda \mathrm{CDM}$ is growing with $z_{\mathrm{SSB}}$ and $\beta$ as the fifth force has more time to operate and is stronger. Comparing runs with the same $\beta$, we see an important effect if the symmetry breaking is earlier. When $z_{\mathrm{SSB}}=2.0$, the fractional power is increasing until we reach a scale where the screening mechanism becomes stronger and then starts to decrease again toward $\Lambda \mathrm{CDM}$, only to start growing again at even smaller scales. This is because the critical density for having screening is much higher for larger $z_{\mathrm{SSB}}$ so that most halos (which are on small scales and of low mass) are unscreened.

In Figure 14 we show the redshift evolution of the power spectrum. The power spectrum is found to be practically identical to that of $\Lambda \mathrm{CDM}$ for redshifts $z>z_{\mathrm{SSB}}$, but as soon as the symmetry breaks at the background level, the symmetron fifth force can kick in and enhance the clustering of matter.

It is clear from Figure 12 that there exist a large range of parameters in which the symmetron model can be distinguished from $\Lambda \mathrm{CDM}$ easily. 

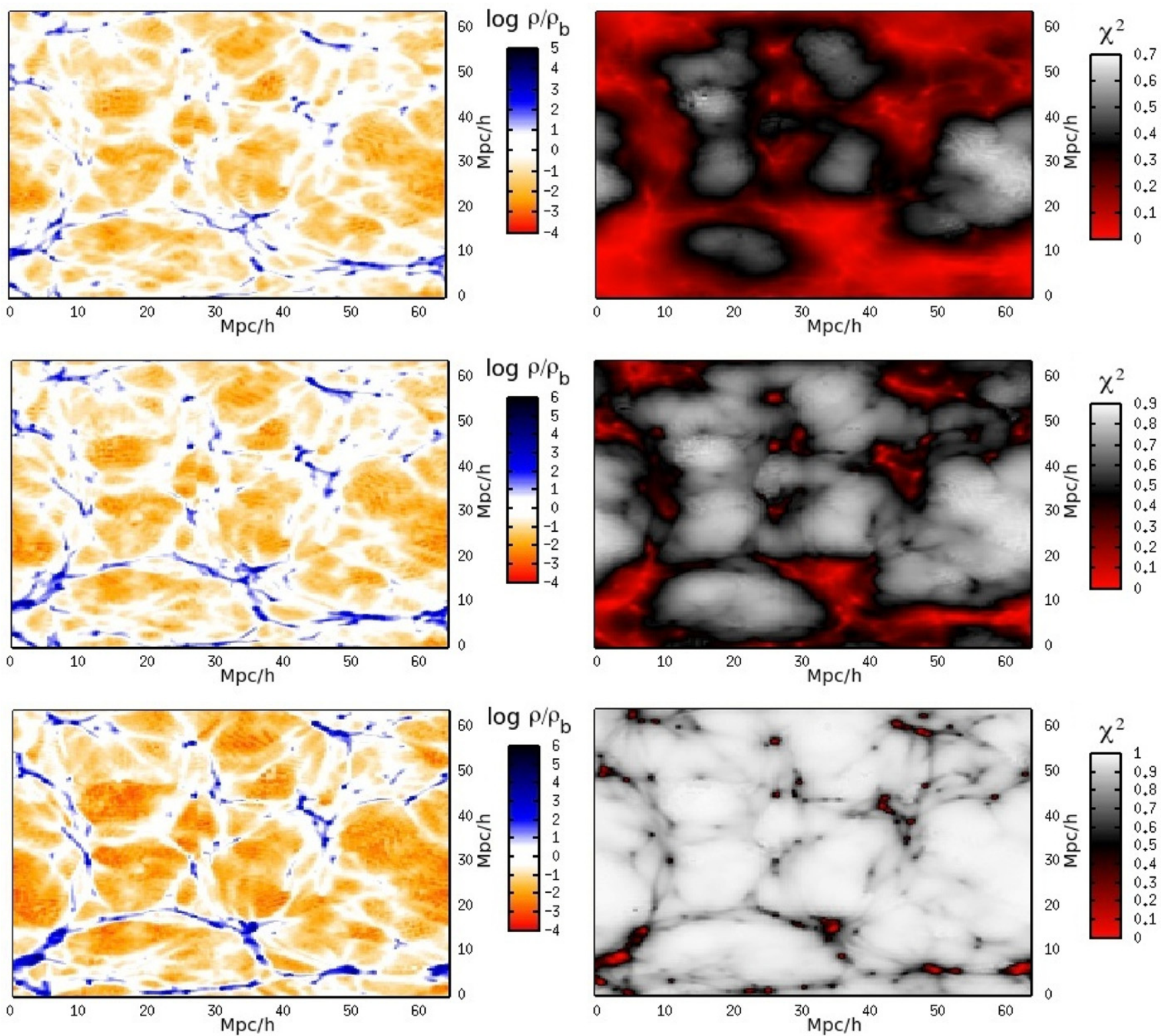

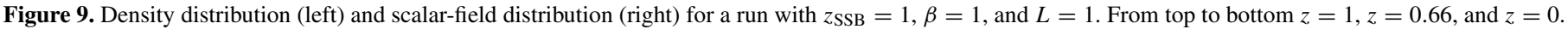
(A color version of this figure is available in the online journal.)

\subsection{Halo Profiles for $\chi$}

In Figure 15 we show the profile of $\chi$ inside the most massive halos found in the simulation. Since the fifth force is proportional to $\chi$, this figure also provides information about the fifth force in halos.

The field profile of $\chi$ is seen to increase from the inner to the outer regions of the halos and shows that the fifth force is most suppressed in the central region as expected.

The fifth force is stronger for smaller halos, because those generally reside in low-density regions where the fifth force is less suppressed. We see that the closer the symmetry breaking redshift is to zero, the smaller $\chi$ becomes inside the halo and the more suppressed the fifth force is. Again this is because early symmetry breaking means a higher critical density and the halo needs to be more massive to be effectively screened. This effect is also seen in Figures 8-10 (note the difference in distribution of $\chi$ at $z=0$ between the different runs) and also on the matter power spectrum in Figure 12.
This has some important consequences for the local constraints. We mentioned in Section 2 that the local constraints were derived by assuming that our galactic neighborhood was not screened today, and lead to the constraint

$$
L\left(1+z_{\mathrm{SSB}}\right)^{3 / 2} \lesssim 2.3
$$

From our numerical results we see that when $z_{\mathrm{SSB}}=2.0$, only the most massive halos are screened. This means that the assumption that went into the constraint above is very likely to be true. On the other hand, for SSB that happens very close to today, halos of much smaller mass are in fact screened and it might be possible to have a range $L$ that exceeds Equation (62) and still be in agreement with experiments.

We note that we have not seen any significant effect on the halo density profiles. For a given mass range, the halo profiles seem to have approximately the same distribution. There should be some important differences for low-mass halos, but the resolution in our simulation is too low to study this. 

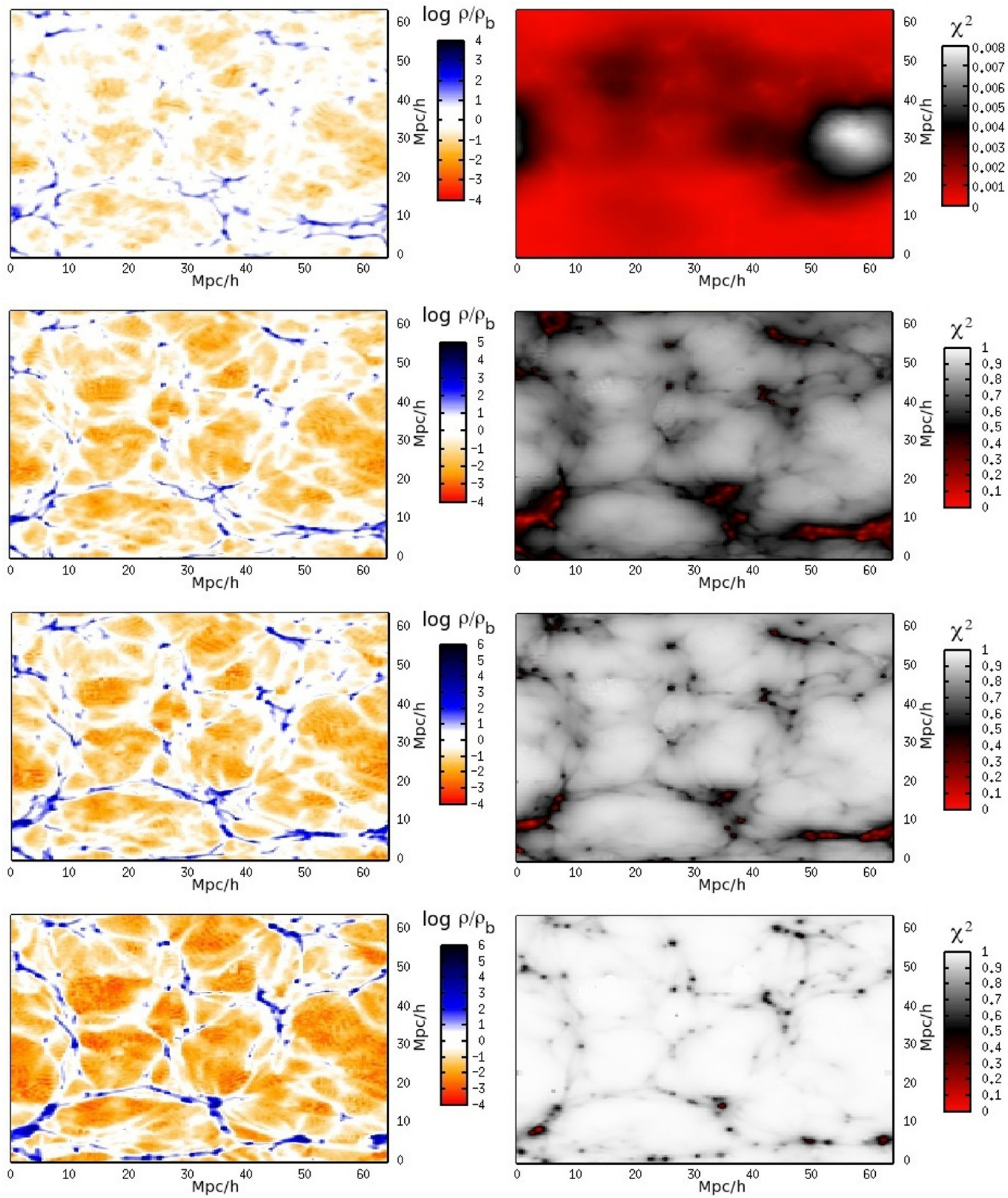

Figure 10. Density distribution (left) and scalar-field distribution (right) for a run with $z_{\mathrm{SSB}}=2, \beta=1$, and $L=1$. From top to bottom $z=2.33, z=1, z=0.66$, and $z=0$.

(A color version of this figure is available in the online journal.)

However, the halo number counts were significantly different as we shall see in the next section.

\subsection{Halo Mass Function}

The halo mass function $n$ is another key structure formation observable. It is defined to be the number density of dark matter halos within a given mass range. Because of the symmetron fifth force, we expect more halos to be formed relative to the standard $\Lambda$ CDM scenario.

We first look at the total number of halos (the integrated mass function) with more than 100 particles, which clearly shows the effect of the fifth force (see Table 2). 


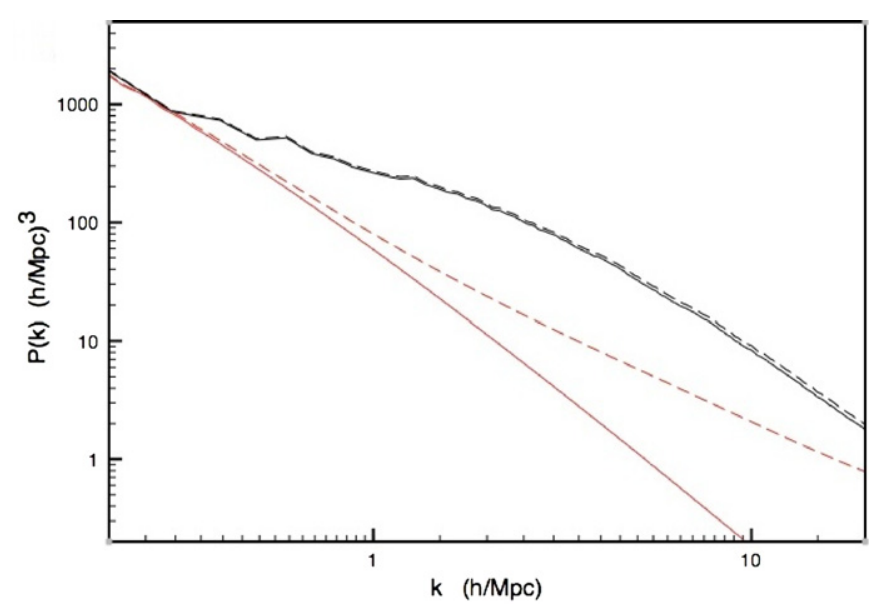

Figure 11. Full nonlinear power spectrum for a run with $z_{\mathrm{SSB}}=L=\beta=1.0$ (dashed black) and $\Lambda \mathrm{CDM}$ (solid black). For comparison we also show the corresponding predictions from linear perturbation theory in red. We clearly see the effectiveness of the screening mechanism. The linear predictions do not take the symmetron mechanism into account and are hugely overestimating the power on small scales relative to $\Lambda \mathrm{CDM}$.

(A color version of this figure is available in the online journal.)

In Figure 16 we have shown the mass function of the symmetron compared to $\Lambda \mathrm{CDM}$ at $z=0$. We see a significantly higher mass function, especially for low-mass halos, which are generally found in low-density regions where the fifth force
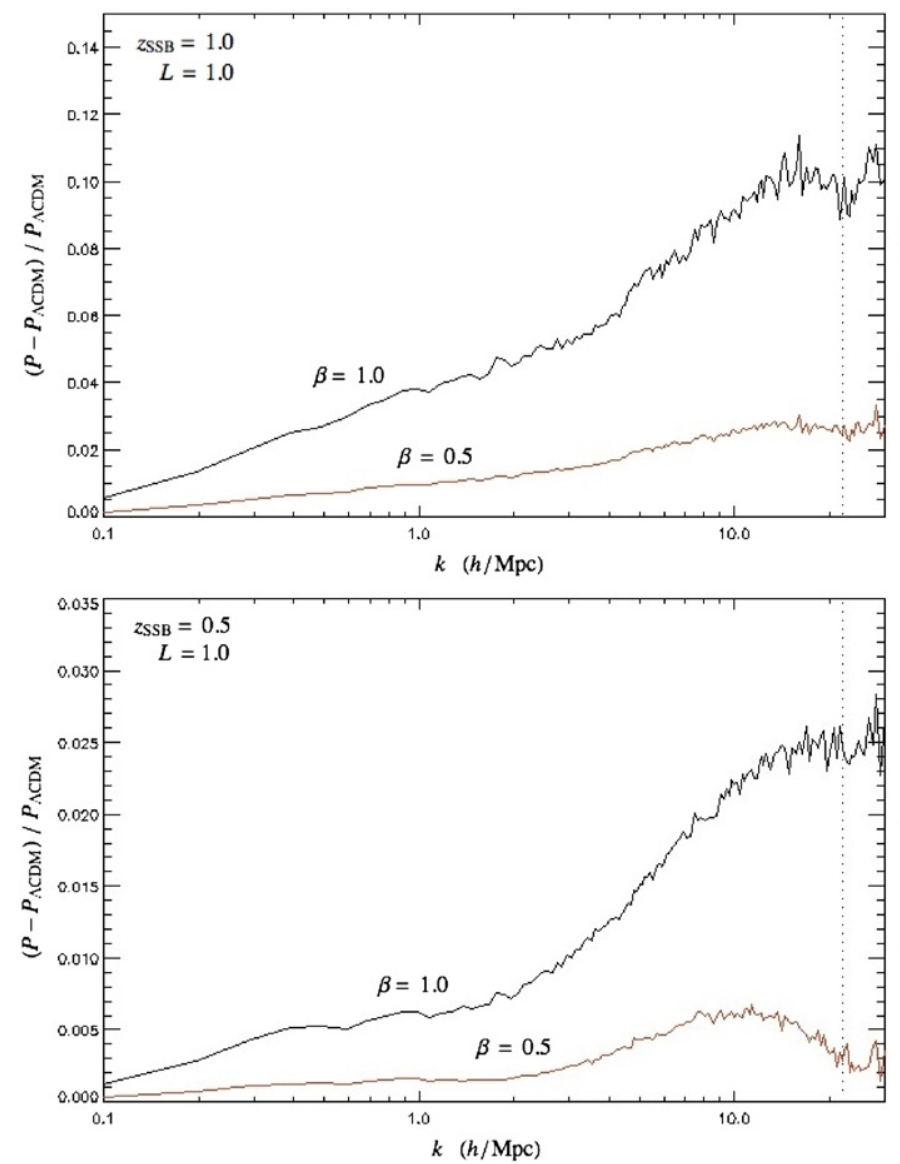

Table 2

The Total Halo Count for Our Nine Simulations at $z=0.0$

\begin{tabular}{lccccccccc}
\hline \hline Simulation & A & B & C & D & E & F & G & H & $\Lambda$ CDM \\
\hline
\end{tabular}

Note. The corresponding symmetron parameters for the runs A-H can be found in Table 1.

is unscreened. The earlier symmetry breaking occurs and the stronger the coupling strength $\beta$, the more halos are formed in agreement to what we would naively expect.

The mass function converges to that of $\Lambda \mathrm{CDM}$ at very large halo masses for most parameters we have looked at. This is because the most massive halos have taken a very long time to form, and therefore when the symmetron kicks in at some low redshift, the halo is already massive enough to be screened. However, for the largest $z_{\mathrm{SSB}}=2.0$ we do have small increases in both the halo number density and the mass of the most massive halos. There have been reports of some tension between observations and $\Lambda \mathrm{CDM}$ predictions with regard to very massive halos. Unfortunately, for the symmetron model to be able to elevate this tension significantly we would need values of the parameters that are in conflict with local experiments.

On the other hand, the symmetron seems to produce a large excess of low-mass halos for some values of the parameters. These are many times the satellite structures of main galactic or
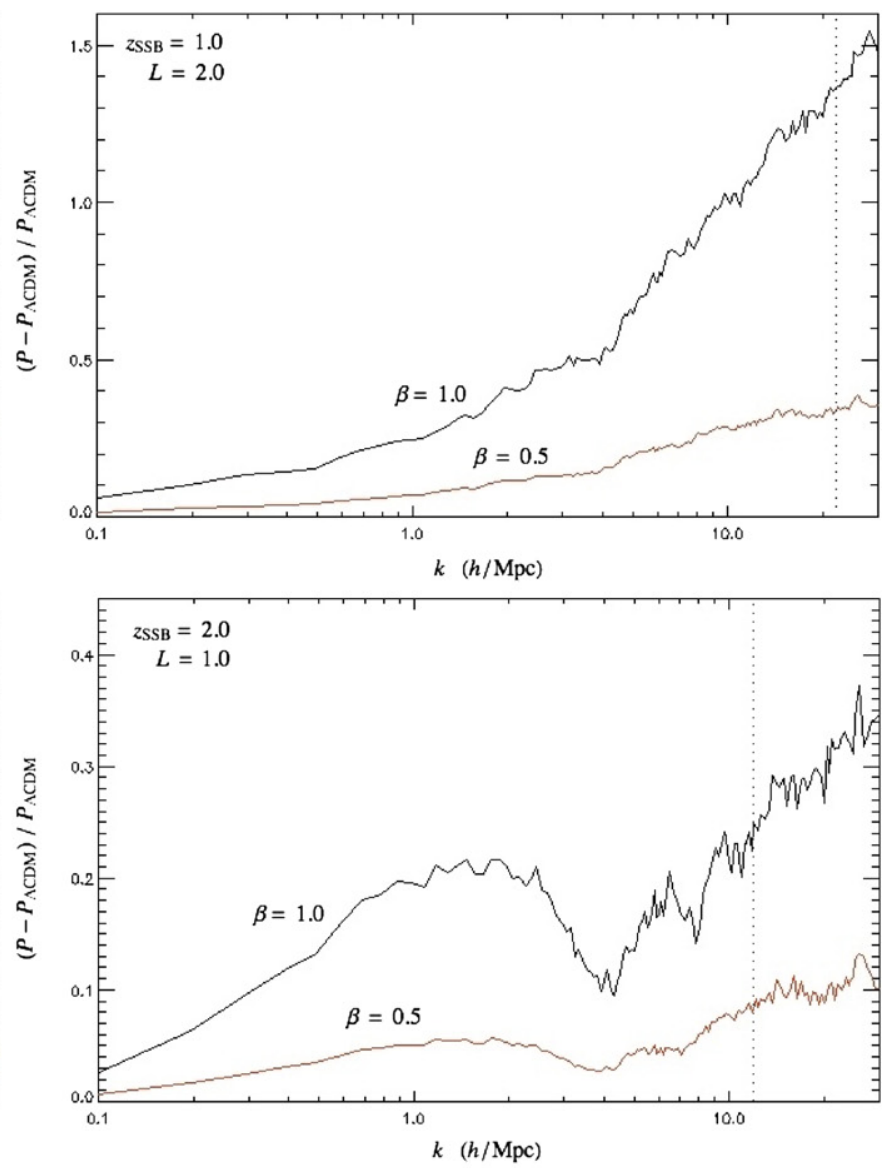

Figure 12. Fractional difference in the nonlinear power spectrum relative to $\Lambda \mathrm{CDM}$ for $\left\{z_{\mathrm{SSB}}=1.0, L=1.0\right\}$ (top left), $\left\{z_{\mathrm{SSB}}=1.0, L=2.0\right\}$ (top right), $\left\{z_{\mathrm{SSB}}=0.5, L=1.0\right\}$ (bottom left), and $\left\{z_{\mathrm{SSB}}=2.0, L=1.0\right\}$ (bottom right). For each case we show the results for the two values $\beta=0.5$ and $\beta=1.0$. The vertical dotted line shows the scale $\min \left(k_{\max }, k_{\mathrm{SN}}\right)$ (see Equation (61)), for which we expect our results to be reliable.

(A color version of this figure is available in the online journal.) 


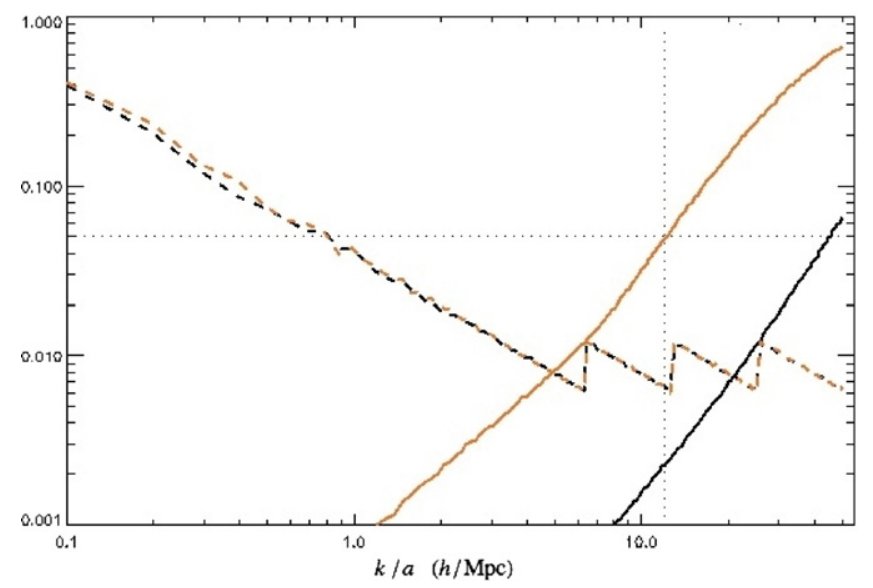

Figure 13. Expected $1 / N_{\text {particles }}$ shot-noise contribution to the power spectrum, $P_{\text {shot-noise }} / P$ (solid), together with the statistical error $\Delta P / P$ in the power spectrum estimation (dashed) for the two redshifts $z=0.0$ (black) and $z=2.0$ (orange) for a simulation with $\left\{z_{\mathrm{SSB}}=2.0, L=1.0, \beta=1.0\right\}$. See Colombi et al. (2009) for details about how the statistical error is computed. The vertical dotted line shows the scale $k_{\mathrm{SN}} \simeq 12 \mathrm{~h} \mathrm{Mpc}^{-1}$ for which the expected shot-noise contribution is $5 \%$ at $z=z_{\mathrm{SSB}}=2.0$.

(A color version of this figure is available in the online journal.)

cluster-sized halos. This increases the long-standing satellite problem of $\Lambda \mathrm{CDM}$, i.e., the lower abundance of satellite structures seen in observations compared to $N$-body simulations. We do not draw any conclusions about this at the present as the resolution for the low-mass halos is relatively low plus baryonic physics has not yet been included in our simulations. This point might prove to be a useful constraint on the symmetron model and more general models with a fifth force in the future.

There is a large range of viable parameters for the symmetron where the mass function deviates significantly from $\Lambda \mathrm{CDM}$.

\section{SUMMARY AND CONCLUSIONS}

The symmetron mechanism is a modification of gravity in which a scalar field is non-minimally coupled to matter, but the screening mechanisms result in potential fifth forces evading local gravity tests. Despite this, the symmetron does affect structure formation. We have investigated this with $N$-body simulations, finding observable signatures in the matter power spectrum and the halo mass function. Whilst the symmetron suffers from the same fine-tuning problems as chameleon models, it has the advantage of looking like a more natural effective theory.

The energy density of the symmetron is too low to contribute to the dark energy, and we must therefore add a cosmological constant to get accelerated expansion of the universe. The background evolution of the symmetron model is simply indistinguishable from that of the $\Lambda$ CDM model.

This degeneracy is broken by the linear perturbations. In particular, we have shown that the linear growth index $\gamma(z, k)$ can have a significant scale and redshift dependence together with a value today that can be distinguished from the $\Lambda \mathrm{CDM}$ prediction for a large part of the parameter space.

The structure formation in the nonlinear regime was investigated by using $N$-body simulations. $N$-body simulations have the advantage over linear theory in its ability of fully capturing the nonlinear environmental dependence of the symmetron field. Our results confirm the expectation that in high-density environments the fifth force becomes screened. Consequently, the key observables such as the nonlinear matter power spectrum are closer to the $\Lambda \mathrm{CDM}$ predictions than expected from a linear analysis.

We found that the symmetron can still produce large observable signatures in both the nonlinear matter power spectrum and the halo mass function, which could in principle be detected by current and near-future cosmological observations such as Euclid.

Note that in the simulations performed in this work, we have treated baryons as dark matter. However, since the symmetron field has a uniform coupling to all matter fields, we expect that all the results will qualitatively remain even after baryons are included. This has to be explicitly checked from simulations. Baryons are known to have a significant effect on small-scale structures, and a natural extension of our work is to study the effects of baryons when a fifth force is present. This is much more computationally expensive and is left for future work.

In conclusion, the symmetron model has been found to have a wide range of observable cosmological effects on both linear and nonlinear scales. This adds to the list of observational signatures like making galaxies brighter (Davis et al. 2011), environment dependence of dark matter halos (Winther et al. 2011), and the possibility of being detected in near-future local gravity experiments (Hinterbichler \& Khoury 2010), to mention some.
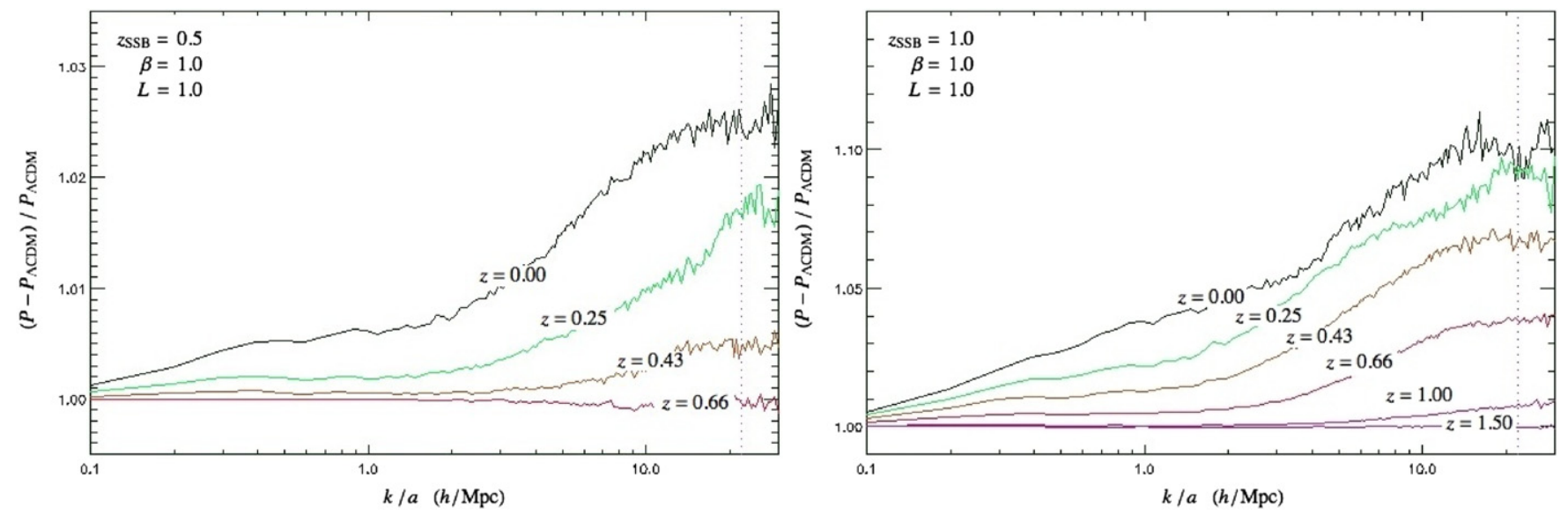

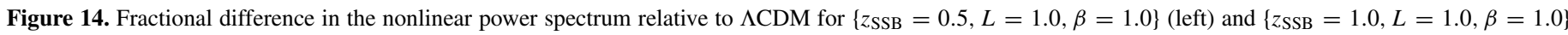
(right) as a function of redshifts. The vertical dotted line shows the scale $\min \left(k_{\max }, k_{\mathrm{SN}}\right)$ (see Equation (61)), for which we expect our results to be reliable. (A color version of this figure is available in the online journal.) 

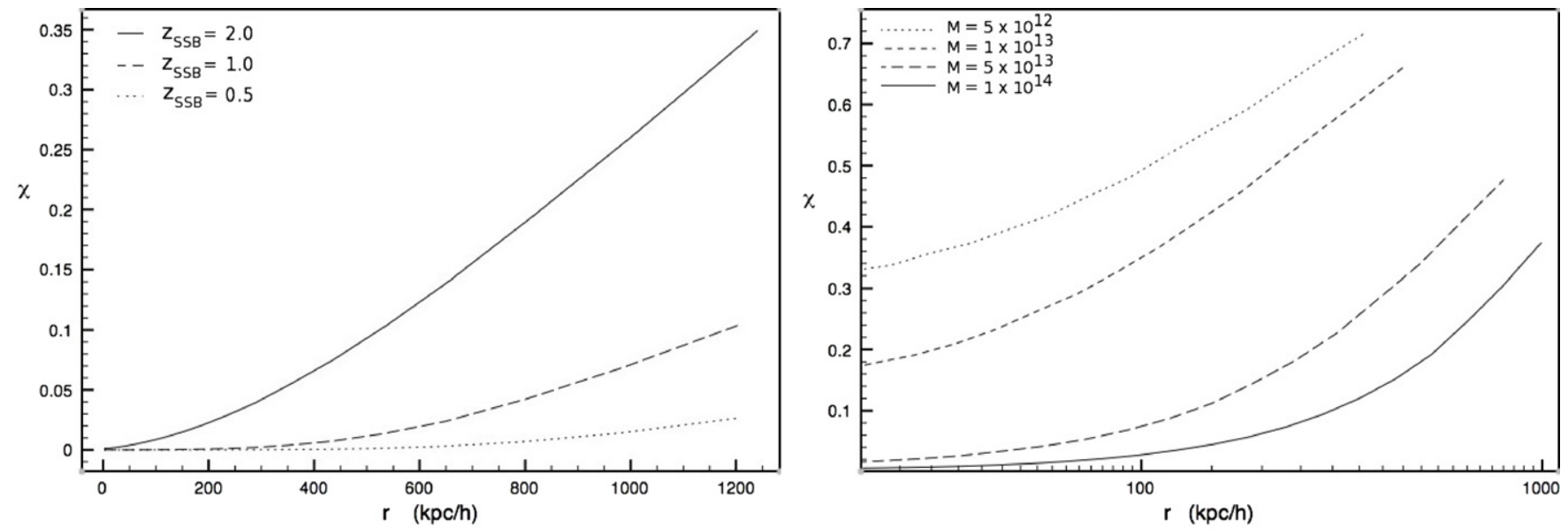

Figure 15. Left: the halo profile of $\chi$ in the most massive halo of the simulation for three different symmetry breaking redshifts. Right: the halo profile of $\chi$ for four halos of mass (from top to bottom) $M=\left\{5 \times 10^{12}, 10^{13}, 5 \times 10^{13}, 10^{14}\right\} M_{\text {sun }} / h$ in the same simulation where $z_{\text {SSB }}=2.0$. In both cases we have fixed $\beta=L=1$.
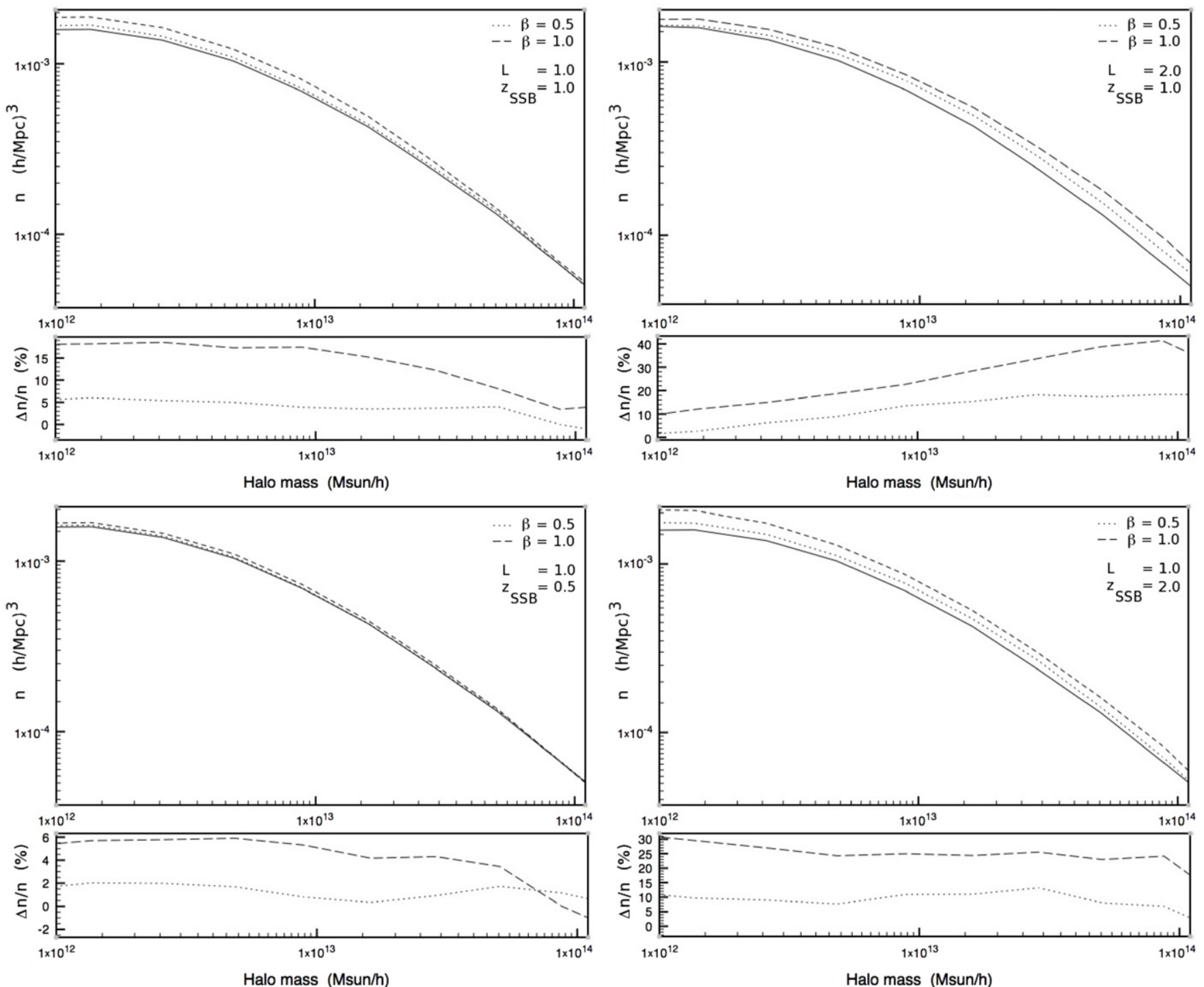

Figure 16. Halo mass function for $\left\{z_{\mathrm{SSB}}=1.0, L=1.0\right\}$ (top left), $\left\{z_{\mathrm{SSB}}=1.0, L=2.0\right\}$ (top right), $\left\{z_{\mathrm{SSB}}=0.5, L=1.0\right\}$ (bottom left), and $\left\{z_{\mathrm{SSB}}=2.0, L=1.0\right\}$ (bottom right). The solid black line shows the prediction of $\Lambda \mathrm{CDM}(\beta=0)$, and the dotted and dashes lines are for the two values $\beta=0.5$ and $\beta=1.0$, respectively. We also show the fractional difference from $\Lambda \mathrm{CDM}$. Note that we have smoothed the mass function over neighboring bins to remove noise arising from the binning to show the trends more clearly. 
The symmetron is therefore a good candidate for the detection of new physics beyond the standard model.

The work described in this paper has been performed on TITAN, the computing facilities at the University of Oslo in Norway. The matter power spectrum was computed using POWMES (Colombi et al. 2009) and the halo properties using MHF (Gill et al. 2004). D.F.M. and H.A.W. thank the Research Council of Norway FRINAT grant $197251 /$ V30. D.F.M. is also partially supported by project PTDC/FIS/111725/2009 and CERN/FP/116398/2010. H.A.W. thanks DAMPT at Cambridge University for the hospitality where a part of this work was carried out. B.L. is supported by Queens' College and the Department of Applied Maths and Theoretical Physics of University of Cambridge. B.L. and A.C.D. thank STFC for partial support. We also thank Douglas Shaw for useful discussions and the anonymous referee for many useful comments that have improved this work.

\section{APPENDIX A}

\section{USEFUL EXPRESSIONS}

Up to first order in the perturbed metric variables $\Xi, \Psi$ the non-zero components of the symmetric Levi-Civita connection are

$$
\begin{gathered}
\Gamma_{00}^{0}=\frac{a^{\prime}}{a}+\Xi^{\prime} \\
\Gamma_{0 k}^{0}=\Xi_{, k} \\
\Gamma_{00}^{i}=\Xi^{, i} \\
\Gamma_{0 k}^{i}=\left(\frac{a^{\prime}}{a}-\Psi^{\prime}\right) \delta_{k}^{i} \\
\Gamma_{j k}^{0}=\delta_{j k}\left(\frac{a^{\prime}}{a}(1-2 \Xi-2 \Psi)-\Psi^{\prime}\right) \\
\Gamma_{j k}^{i}=-\Psi_{, k} \delta_{j}^{i}-\Psi,{ }_{j} \delta_{k}^{i}+\Psi^{i} \delta_{j k} .
\end{gathered}
$$

From these expressions we find that the components of the Ricci tensor and Ricci scalar are found to be

$$
\begin{aligned}
& R_{00}=\Xi{ }_{i} \\
&+-3\left(\frac{a^{\prime \prime}}{a}-\left(\frac{a^{\prime}}{a}\right)^{2}\right)+3 \Psi^{\prime \prime} \\
&+3 \frac{a^{\prime}}{a}\left(\Psi^{\prime}+\Xi^{\prime}\right) \\
& R_{0 j}=2 \Psi_{, j}^{\prime}+2 \frac{a^{\prime}}{a} \Xi_{, j} \\
& R_{i j}=-\Psi^{\prime \prime} \delta_{i j}-\frac{a^{\prime}}{a}\left(\Xi^{\prime}+5 \Psi^{\prime}\right) \delta_{i j}-\Psi_{, k}^{k} \delta_{i j} \\
&+\left(\frac{a^{\prime \prime}}{a}+\left(\frac{a^{\prime}}{a}\right)^{2}\right)\left(1-2 \Psi-2 \Xi^{2} \delta_{i j}\right. \\
&-(\Xi-\Psi), i j \\
& R=6 \frac{a^{\prime \prime}}{a^{3}}(1-2 \Xi)+\frac{1}{a^{2}}\left(4 \Psi_{, k}^{k}-\Xi_{, k}^{k}\right) \\
&-\frac{6}{a^{2}}\left(\Psi \Psi^{\prime \prime}+\frac{a^{\prime}}{a}\left(\Xi^{\prime}+3 \Psi^{\prime}\right)\right) .
\end{aligned}
$$

\section{APPENDIX B}

\section{DISCRETIZATION OF EQUATIONS}

To implement the nonrelativistic equations into our numerical code, we have to rewrite them using code units, which are given by

$$
\begin{aligned}
& \mathbf{x}_{\mathbf{c}}=\frac{\mathbf{x}}{B}, \mathbf{p}_{\mathbf{c}}=\frac{\mathbf{p}}{H_{0} B}, t_{c}=t H_{0}, \chi=\frac{\phi}{\phi_{0}} \\
& \boldsymbol{\Phi}_{\mathbf{c}}=\frac{\boldsymbol{\Phi}}{\left(H_{0} B\right)^{2}}, \rho_{\mathbf{c}}=\frac{\rho_{\mathbf{m}}}{\overline{\rho_{\mathbf{m}}}}, \nabla=\mathbf{B} \nabla_{\mathbf{x}},
\end{aligned}
$$

where subscript $c$ stands for code units, $B$ is the box size, $H_{0}=100 \mathrm{~km} \mathrm{~s}^{-1} \mathrm{Mpc}^{-1}$, and an overline denotes background quantities. In what follows we shall write $\nabla=\nabla_{c}$ for simplicity.

\section{B.1. Scalar Field Equation of Motion}

The equation of motion for $\chi$ in code units becomes

$$
\begin{aligned}
\frac{a c^{2}}{\left(B H_{0}\right)^{2}} \nabla^{2} \chi & \simeq a^{3}\left(\bar{\chi}-\chi+\chi^{3}-\bar{\chi}^{3}\right)\left(\frac{\mu}{H_{0}}\right)^{2} \\
& +3 \Omega_{m}\left(\frac{M_{\mathrm{pl}}}{M}\right)^{2}\left(\rho_{c} \chi-\bar{\chi}\right),
\end{aligned}
$$

where $\bar{\chi}$ is the background solutions and we have used $\phi_{0}^{2}=$ $\mu^{2} / \lambda$ to simplify. Note that $\chi$ varies in the region $0 \leqslant \chi^{2} \leqslant 1$. Discretized this equation becomes $L^{h}\left(\chi_{i, j, k}\right)=0$, where

$$
\begin{aligned}
L^{h}\left(\chi_{i, j, k}\right)= & \frac{1}{h^{2}} \frac{a c^{2}}{\left(B H_{0}\right)^{2}}\left(\chi_{i+1, j, k}-2 \chi_{i, j, k}+\chi_{i-1, j, k}\right) \\
& +\frac{1}{h^{2}} \frac{a c^{2}}{\left(B H_{0}\right)^{2}}\left(\chi_{i, j+1, k}-2 \chi_{i, j, k}+\chi_{i, j-1, k}\right) \\
& +\frac{1}{h^{2}} \frac{a c^{2}}{\left(B H_{0}\right)^{2}}\left(\chi_{i, j, k+1}-2 \chi_{i, j, k}+\chi_{i, j, k-1}\right) \\
& -a^{3}\left(\frac{\mu}{H_{0}}\right)^{2}\left(\bar{\chi}-\chi_{i, j, k}\right) \times \\
& \times\left(1-\chi_{i, j, k}^{2}-\bar{\chi} \chi_{i, j, k}-\bar{\chi}^{2}\right) \\
& -3 \Omega_{m}\left(\frac{M_{\mathrm{pl}}}{M}\right)^{2}\left(\rho_{c} \chi_{i, j, k}-\bar{\chi}\right) .
\end{aligned}
$$

The Newton-Gauss-Seidel iteration says that we can obtain a new and more accurate solution of $\chi_{i, j, k}^{\text {new }}$ using our knowledge about the old solution $\chi_{i, j, k}^{\text {old }}$ as

$$
\chi_{i, j, k}^{\text {new }}=\chi_{i, j, k}^{\text {old }}-\frac{L^{h}\left(\chi_{i, j, k}^{\text {old }}\right)}{\partial L^{h}\left(\chi_{i, j, k}^{\text {old }}\right) / \partial \chi_{i, j, k}^{\text {old }}}
$$

where

$$
\begin{aligned}
\frac{\partial L^{h}\left(\chi_{i, j, k}\right)}{\partial \chi_{i, j, k}}= & -\frac{6}{h^{2}} \frac{a c^{2}}{\left(B H_{0}\right)^{2}}+a^{3}\left(\frac{\mu}{H_{0}}\right)^{2}\left(1-3 \chi_{i, j, k}^{2}\right) \\
& -3 \Omega_{m}\left(\frac{M_{\mathrm{pl}}}{M}\right)^{2} \rho_{c}
\end{aligned}
$$

\section{B.2. Poisson Equation}

Since we can neglect the scalar field contribution to the Poisson equation, it remains unmodified from that of $\Lambda \mathrm{CDM}$ and reads (in code units)

$$
\nabla^{2} \Phi_{c}=\frac{3}{2} \Omega_{m}\left(\rho_{c, i, j, k}-1\right) .
$$




\section{B.3. Particle Equation of Motion}

Using the code units, Equation (56) can be rewritten as

$$
\begin{gathered}
\frac{d \mathbf{x}_{\mathbf{c}}}{d t_{c}}=\frac{\mathbf{p}_{\mathbf{c}}}{a^{2}} \\
\frac{d \mathbf{p}_{\mathbf{c}}}{d t_{c}}=-\frac{1}{a} \nabla \Phi_{c}-\chi\left(\frac{\beta M}{M_{\mathrm{pl}}}\right)^{2}\left(\frac{c^{2} \nabla \chi}{\left(B H_{0}\right)^{2}}+\frac{d \chi}{d t_{c}} \mathbf{p}_{\mathbf{c}}\right) .
\end{gathered}
$$

The factor $\left(M / M_{\mathrm{pl}}\right)^{2}$ can be also rewritten in terms of $L, \beta$, and $z_{\text {SSB }}$ by using Equation (22).

\section{REFERENCES}

Accetta, F. S., Krauss, L. M., \& Romanelli, P. 1990, Phys. Lett. B, 248, 146 Adelberger, E. G. 2002, in CPT and Lorentz Symmetry, ed. V. A. Kostelecký (Singapore: World Scientific), 9

Appleby, S. A., \& Weller, J. 2010, J. Cosmol. Astropart. Phys., JCAP12(2010)006

Arkani-Hamed, N., Georgi, H., \& Schwartz, M. D. 2003, Ann. Phys., 305, 96

Baldi, M. 2009, Nucl. Phys. B, 194, 178

Baldi, M. 2011, MNRAS, 411, 1077

Baldi, M., Pettorino, V., Robbers, G., \& Springel, V. 2010, MNRAS, 403, 1684

Bertotti, B., Iess, L., \& Tortora, P. 2003, Nature, 425, 374

Bertschinger, E. 2001, ApJS, 137, 1

Binétruy, P. 2006, Supersymmetry: Theory, Experiment, and Cosmology (Oxford, UK: Oxford Univ. Press), 520

Brax, P., Burrage, C., \& Davis, A.-C. 2011a, J. Cosmol. Astropart. Phys., JCAP09(2011)020

Brax, P., van de Bruck, C., Davis, A.-C., et al. 2011b, Phys. Rev. D, 84, 123524

Brax, P., van de Bruck, C., Davis, A.-C., Khoury, J., \& Weltman, A. 2004, Phys. Rev. D, 70, 123518

Brax, P., van de Bruck, C., Davis, A.-C., Li, B., \& Shaw, D. J. 2011c, Phys. Rev. D, 83, 104026

Brax, P., van de Bruck, C., Davis, A.-C., Mota, D. F., \& Shaw, D. J. 2007a, Phys. Rev. D, 76, 124034

Brax, P., van de Bruck, C., Davis, A.-C., Mota, D. F., \& Shaw, D. J. 2007b, Phys. Rev. D, 76, 085010

Brax, P., van de Bruck, C., Davis, A.-C., \& Shaw, D. J. 2008, Phys. Rev. D, 78, 104021

Brax, P., van de Bruck, C., Davis, A.-C., \& Shaw, D. 2010a, Phys. Rev. D, 82, 063519

Brax, P., van de Bruck, C., Davis, A.-C., \& Shaw, D. 2010b, J. Cosmol. Astropart. Phys., JCAP04(2010)032

Brax, P., van de Bruck, C., Mota, D. F., Nunes, N. J., \& Winther, H. A. 2010c, Phys. Rev. D, 82, 083503

Clampitt, J., Jain, B., \& Khoury, J. 2012, J. Cosmol. Astropart. Phys., JCAP01(2012)030

Clifton, T., Mota, D. F., \& Barrow, J. D. 2005, MNRAS, 358, 601

Colombi, S., Jaffe, A., Novikov, D., \& Pichon, C. 2009, MNRAS, 393, 511

Davis, A.-C., Lim, E. A., Sakstein, J., \& Shaw, D. 2011, arXiv:1102.5278

de Rham, C. 2010, Phys. Lett. B, 688, 137 de Rham, C., Dvali, G., Hofmann, S., et al. 2008, Phys. Rev. Lett., 100, 251603 Decca, R. S., López, D., Fischbach, E., et al. 2007, Phys. Rev. D, 75, 077101 Deffayet, C., Dvali, G., Gabadadze, G., \& Vainshtein, A. 2002, Phys. Rev. D, 65,044026

Dvali, G., Gabadadze, G., \& Porrati, M. 2000, Phys. Lett. B, 485, 208

Felder, G., Kofman, L., \& Linde, A. 2001, Phys. Rev. D, 64, 123517

Ferraro, S., Schmidt, F., \& Hu, W. 2011, Phys. Rev. D, 83, 063503

Gabadadze, G. 2009, Phys. Lett. B, 681, 89

Gannouji, R., Moraes, B., Mota, D. F., et al. 2010, Phys. Rev. D, 82, 124006

Gies, H., Mota, D. F., \& Shaw, D. J. 2008, Phys. Rev. D, 77, 025016

Gill, S. P. D., Knebe, A., \& Gibson, B. K. 2004, MNRAS, 351, 399

Hellwing, W. A., \& Juszkiewicz, R. 2009, Phys. Rev. D, 80, 083522

Hellwing, W. A., Juszkiewicz, R., \& van de Weygaert, R. 2010, Phys. Rev. D, 82,103536

Hinterbichler, K., \& Khoury, J. 2010, Phys. Rev. Lett., 104, 231301

Hinterbichler, K., Khoury, J., Levy, A., \& Matas, A. 2011, Phys. Rev. D, 84 103521

Hinterbichler, K., Trodden, M., \& Wesley, D. 2010, Phys. Rev. D, 82, 124018

Hoskins, J. K., Newman, R. D., Spero, R., \& Schultz, J. 1985, Phys. Rev. D, 32, 3084

Hwang, J.-C., \& Noh, H. 2002, Phys. Rev. D, 65, 023512

Khoury, J. 2010, arXiv:1011.5909

Khoury, J., \& Weltman, A. 2004, Phys. Rev. D, 69, 044026

Knebe, A., Green, A., \& Binney, J. 2001, MNRAS, 325, 845

Li, B., \& Barrow, J. D. 2011a, Phys. Rev. D, 83, 024007

Li, B., \& Barrow, J. D. 2011b, MNRAS, 413, 262

Li, B., Mota, D. F., \& Barrow, J. D. 2011a, ApJ, 728, 109

Li, B., Mota, D. F., \& Barrow, J. D. 2011b, ApJ, 728, 108

Li, B., \& Zhao, H. 2010, Phys. Rev. D, 81, 104047

Linde, A. 2008, in Inflationary Cosmology, ed. M. Lemoine, J. Martin, \& P. Peter (Lecture Notes in Physics, Vol. 738; Berlin: Springer), 1

Mota, D. F., \& Barrow, J. D. 2004a, MNRAS, 349, 291

Mota, D. F., \& Barrow, J. D. 2004b, Phys. Lett. B, 581, 141

Mota, D. F., Sandstad, M., \& Zlosnik, T. 2010, J. High Energy Phys., JHEP12(2010)051

Mota, D. F., \& Shaw, D. J. 2006, Phys. Rev. Lett., 97, 151102

Mota, D. F., \& Shaw, D. J. 2007, Phys. Rev. D, 75, 063501

Mota, D. F., \& Winther, H. A. 2011, ApJ, 733, 7

Motohashi, H., Starobinsky, A. A., \& Yokoyama, J. 2010, Prog. Theor. Phys., 123,887

Nagata, R., Chiba, T., \& Sugiyama, N. 2004, Phys. Rev. D, 69, 083512

Narikawa, T., \& Yamamoto, K. 2010, Phys. Rev. D, 81, 043528

Nicolis, A., Rattazzi, R., \& Trincherini, E. 2009, Phys. Rev. D, 79, 064036

Olive, K. A., \& Pospelov, M. 2008, Phys. Rev. D, 77, 043524

Oyaizu, H., Lima, M., \& Hu, W. 2008, Phys. Rev. D, 78, 123524

Prunet, S., Pichon, C., Aubert, D., et al. 2008, ApJS, 178, 179

Schmidt, F. 2009, Phys. Rev. D, 80, 043001

Schmidt, F. 2010, Phys. Rev. D, 81, 103002

Schmidt, F., Lima, M., Oyaizu, H., \& Hu, W. 2009, Phys. Rev. D, 79, 083518

Stabenau, H. F., \& Jain, B. 2006, Phys. Rev. D, 74, 084007

Tsujikawa, S., Gannouji, R., Moraes, B., \& Polarski, D. 2009, Phys. Rev. D, 80 , 084044

Vainshtein, A. I. 1972, Phys. Lett. B, 39, 393

Winther, H. A., Mota, D. F., \& Li, B. 2011, arXiv:1110.6438

Zhao, G.-B., Li, B., \& Koyama, K. 2011, Phys. Rev. D, 83, 044007

Zhao, H., Macciò, A. V., Li, B., Hoekstra, H., \& Feix, M. 2010, ApJ, 712, L179 